\title{
New differential equations for on-shell loop integrals
}

\author{
James M. Drummond, ${ }^{a, b}$ Johannes M. Henn ${ }^{c}$ and Jaroslav Trnka ${ }^{d, e}$ \\ ${ }^{a}$ PH-TH Division, CERN, \\ Geneva, Switzerland \\ ${ }^{b}$ LAPTH, Université de Savoie, CNRS, \\ B.P. 110, F-74941 Annecy-le-Vieux Cedex, France \\ ${ }^{c}$ Institut für Physik, Humboldt-Universität zu Berlin, \\ Newtonstraße 15, D-12489 Berlin, Germany \\ ${ }^{d}$ School of Natural Sciences, Institute for Advanced Study, \\ Princeton, NJ 08540, U.S.A. \\ ${ }^{e}$ Department of Physics, Princeton University, \\ Princeton, NJ 08540, U.S.A. \\ E-mail: drummond@lapp.in2p3.fr, henn@physik.hu-berlin.de, \\ jtrnka@ias.edu
}

ABSTRACT: We present a novel type of differential equations for on-shell loop integrals. The equations are second-order and importantly, they reduce the loop level by one, so that they can be solved iteratively in the loop order. We present several infinite series of integrals satisfying such iterative differential equations. The differential operators we use are best written using momentum twistor space. The use of the latter was advocated in recent papers discussing loop integrals in $\mathcal{N}=4$ super Yang-Mills. One of our motivations is to provide a tool for deriving analytical results for scattering amplitudes in this theory. We show that the integrals needed for planar MHV amplitudes up to two loops can be thought of as deriving from a single master topology. The master integral satisfies our differential equations, and so do most of the reduced integrals. A consequence of the differential equations is that the integrals we discuss are not arbitrarily complicated transcendental functions. For two specific two-loop integrals we give the full analytic solution. The simplicity of the integrals appearing in the scattering amplitudes in planar $\mathcal{N}=4$ super Yang-Mills is strongly suggestive of a relation to the conjectured underlying integrability of the theory. We expect these differential equations to be relevant for all planar MHV and non-MHV amplitudes. We also discuss possible extensions of our method to more general classes of integrals.

KEywords: Supersymmetric gauge theory, Integrable Equations in Physics, Conformal and W Symmetry, 1/N Expansion 


\section{Contents}

1 Introduction 2

2 Motivation: MHV amplitudes $\quad 5$

3 Differential equations for loop integrals $\quad 9$

3.1 Finite integrals 9

3.1.1 Ladders 9

$\begin{array}{lll}3.1 .2 & \text { Pentaladders } & 11\end{array}$

$\begin{array}{lll}3.1 .3 & \text { Pentaladders reloaded } & 14\end{array}$

$\begin{array}{ll}\text { 3.1.4 Double pentaladders } & 18\end{array}$

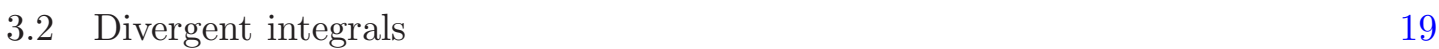

$\begin{array}{lll}3.2 .1 & \text { One-loop box integrals } & 19\end{array}$

$\begin{array}{lll}3.2 .2 & \text { Penta-box integrals } & 20\end{array}$

4 Solving the differential equations $\quad 22$

4.1 Harmonic polylogarithms 22

4.2 Solution for seven-point pentagon and penta-box integral 23

4.3 Equations for eight-point pentagon and pentaladder integrals 26

4.4 Equations for the six-point hexagon and double-pentagon integrals 28

$\begin{array}{llr}5 & \text { Conclusion and outlook } & 29\end{array}$

A Twistor differential operators $\quad 30$

$\begin{array}{lll}\text { A.1 Diagrams in the one-loop amplitude } & 30\end{array}$

A.1.1 Two-mass hard diagram 31

A.1.2 Pentagon diagram 32

A.1.3 Differential equations for the one-loop MHV amplitude 33

A.2 Diagrams in the two-loop amplitude 33

A.2.1 Double-boxes 33

A.2.2 Pentagon-boxes 34

A.2.3 Double-pentagons 34

B Five-point pentagon and penta-box integrals $\quad 35$ 


\section{Introduction}

In this paper we present new differential equations for on-shell loop integrals. Our main motivation is to develop efficient methods to determine the loop-level S-matrix in $\mathcal{N}=4$ super Yang-Mills (SYM), which is built from precisely the types of integrals we discuss here. The planar $\mathcal{N}=4$ theory is believed to be governed by some underlying integrable structure and hence it certainly deserves to have a beautiful and simple S-matrix. Our differential equations can be viewed as a step towards explaining this simplicity, as they restrict the integrals contributing to the amplitudes. Our results are at the level of specific integrals and so can also be used for subsets of integrals appearing in less supersymmetric theories. We also expect our method to be applicable to larger classes of integrals.

The simplicity of the loop integrals implied by the differential equations we find is suggestive of a connection to the expected underlying integrability of planar $\mathcal{N}=4 \mathrm{SYM}$, and more concretely, of the underlying symmetries of its tree-level amplitudes. It was found that the tree-level S-matrix in this theory has a hidden symmetry, dual superconformal symmetry [1-3]. Together with the ordinary superconformal symmetry of the theory, it leads to a Yangian symmetry [4]. At loop level, a priori the symmetries are broken.

The breaking of the bosonic part of dual superconformal symmetry is well understood and is controlled by anomalous Ward identities $[1,5]$ that were initially derived for certain Wilson loops, and are conjectured to hold for the scattering amplitudes. The Ward identities completely fix the functional form of the four-point and five-point amplitudes, confirming the ABDK/BDS conjecture for these cases $[6,7]$. Starting from six points, a modification is required, called the remainder function $[8,9]$. An alternative way of understanding the dual conformal symmetry at loop level is possible by using the massive regulator of [10], which is inspired by the AdS/CFT correspondence [11]. This setup is expected to make dual conformal symmetry exact, i.e. unbroken, at loop level. Given this, it is natural to assume that the loop-level integral basis of $\mathcal{N}=4 \mathrm{SYM}$ should consist of integrals having this exact symmetry (see [12] and references therein). Indeed, the absence of triangle sub-graphs at one loop was confirmed in [13] and further support for this conjecture comes from [14].

A similar understanding of the full Yangian symmetry at loop level is still lacking and subject to ongoing research [15-18]. Recently it was argued that the (unregulated) loop integrand has the full Yangian symmetry, up to total derivatives [19]. Indeed the integrand can be recursively constructed via a BCFW type recursion relation [19] (see also [20]). Ignoring regularisation, this recursive construction can be written as a sequence of Yangian invariant operations on the basic Yangian invariant functions [19] (see also [2124] for a discussion of Yangian invariants).

Just as the dual conformal symmetry of the integrand [25] was a hint that there is an anomalous Ward identity [5], the existence of the Yangian invariant integrand indicates that there should be a way to directly understand the breaking of the full symmetry. Although we do not yet make a direct connection to the Yangian generators, we find it likely that the underlying Yangian symmetry is related to the differential equations we find.

Further very concrete motivation for our study also comes from recent explicit results 
for the hexagonal light-like Wilson loop, which is dual to the six-gluon MHV amplitude [8, 9] (for reviews see [26, 27] and references therein, and [28, 29] for recent developments). In [30], a remarkably simple form of the six-point remainder function $[8,9]$ was given, based on previous work [31]. Simple results are also conjectured to hold in special kinematics [32, 33]. Recently, the analytic six-point Wilson loop result of [31] could be reproduced in a kinematical limit by an analytic calculation of the corresponding scattering amplitude, extending previous calculations for mass-regulated amplitudes [12, 34]. Related recent work on loop amplitudes in $\mathcal{N}=4 \mathrm{SYM}$ can be found in $[35,36]$. See also [37, 38] for other related recent developments.

The use of differential equations to evaluate loop integrals [39-41] is widespread in the literature, for a review see chapter 7 of [42]. In this approach one considers a set of master integrals, and an associated family of integrals is be obtained from by shrinking lines. One differentiates the master integrals with respect to kinematical invariants or masses. The result is in general a linear combination of several integrals. Integral reduction identities are then applied to re-express the latter in terms of the set of master integrals. In complicated cases this step can be non-trivial, as in general it requires the knowledge of all reduction identities. In general one obtains a set of first-order differential equations for the master integrals. A disadvantage is that in order to solve for a given integral, one has to deal with all integrals of a given family. It can also happen that when computing a finite integral, intermediate steps contain divergences that only cancel at the end, and a regulator has to be used.

The differential equations we obtain here are quite different in nature. There are two important differences to the method described above: Firstly, our differential equations can be applied directly to a given integral, without having to know all integrals with fewer propagators, or any integral reduction identities. Secondly, they are second-order equations, and, importantly, they reduce the loop level by one. In other words, the homogeneous term of the equations corresponds to lower-loop integrals, and the equations therefore have an iterative structure.

While the differential equations method known in the literature can be applied in principle to all loop integrals, the differential equations we find are specific to a certain class of integrals. The latter constitute a subset of integrals needed for computing scattering amplitudes in a generic theory. There is reason to believe that in the special case of $\mathcal{N}=4$ SYM, all planar loop integrals are constrained by the type of differential equations we find here. Very concretely we observe that the one- and two-loop MHV amplitudes in $\mathcal{N}=4 \mathrm{SYM}$ in the form given in [19], can all be expressed in terms of a single 'master' integral, namely
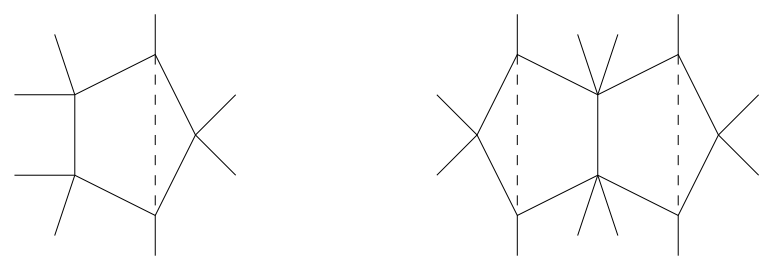

at one- and two-loop order, respectively. The reason is that reduced topologies, such as box, double box or penta-box topologies, can be obtained by taking soft limits of the master 
integrals. We will give the precise definition of the integrals later. In the six-point case, they were used recently to obtain an analytic result for the remainder function at two loops (in a kinematical limit) by two of the present authors in [43]. This is the first time that such an analytical result was obtained directly for the scattering amplitude, and the results also hinted at a certain simplicity of the integrals that had to be computed. We will make this more precise in this paper. As we will show, the integrals depicted in (1.1) satisfy second-order differential equations that reduce their loop degree by one, namely

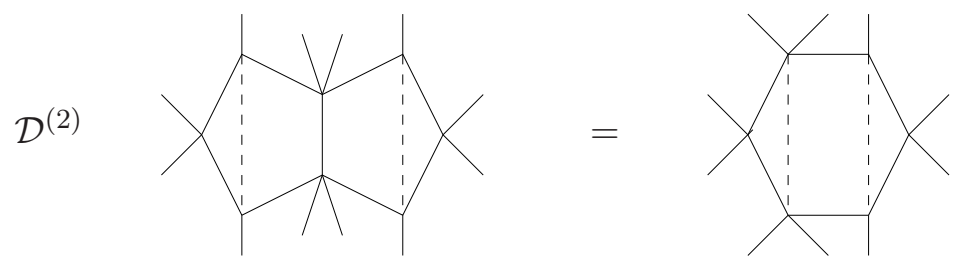

The one-loop integrals appearing in (1.1) and on the r.h.s. of (1.2) satisfies a similar equation with simple rational functions as inhomogeneous terms. These equations imply powerful constraints on the functional dependence of those integrals. In the following we will give several examples where we solve such equations and obtain analytic answers for two-loop integrals with multiple external legs.

Iterative differential equations of the type (1.2) were previously found in [25] by two of the present authors and Smirnov and Sokatchev for certain off-shell ladder (i.e. multiple box) integrals, and are closely related to the equations we find here. We will review the differential equations of [25] in section 3. The differential equations presented here can be thought of as a generalisation of the ones used in [25] to on-shell integrals.

We find it very convenient to work with momentum twistor variables [44]. These are well-suited to describe planar loop integrals. They solve the momentum conservation and on-shell constraints and therefore are unconstrained variables. This makes them the natural variables to use if one is interested in differential equations. One-loop box integrals were discussed in momentum twistor space in $[45,46]$. In the cases where infrared divergences are present we employ the AdS-inspired mass regulator of [10]. The latter allows us to stay in four dimensions and continue to use momentum twistors in those cases as well. For recent references using momentum twistors and this regulator see [43, 45, 46].

The outline of the paper is as follows. In section 2, we recall the definition of momentum twistor variables and the expression for the one- and two-loop MHV amplitudes in $\mathcal{N}=4$ SYM. It is shown that at each loop level there is only one 'master' topology. In section 3 we show that certain classes of integrals satisfy second-order differential equations that reduce the loop order by one. We first review the differential equations for off-shell integrals found in [25], and then generalise them to the on-shell case. We present two mechanisms for finding such differential equations. We present several infinite classes of integrals satisfying iterative differential equations. In section 4 we give an example for how to solve the differential equations, using certain assumptions about boundary conditions. We give explicit analytical results for several multi-leg integrals at two loops. In section 5 we conclude and comment on several possibilities of extending our method. There are two appendices. In appendix A we discuss twistor differential operators that annihilate 
the integrand of the integrals appearing in the MHV amplitudes, up to anomalies. Appendix B contains the analytic formulas for the one- and two-loop penta-box integrals with "magic" numerator.

\section{Motivation: MHV amplitudes}

In this section we recall how momentum twistor variables can be used to describe loop integrals. We also recall the recently-proposed integral representation for planar two-loop MHV amplitudes in $\mathcal{N}=4$ super Yang-Mills [19]. We show that at each loop order, all integrals contained in the MHV amplitudes can be thought of as deriving from a single master topology. Reduced topologies are obtained by taking soft limits. This is related to the consistency of the loop integrand with soft limits [43].

Let us briefly recall how the momentum twistor variables are related to the standard momentum space variables. Given the $n$ incoming light-like momenta of a planar colourorderd ordered amplitude,

$$
p_{i}^{\alpha \dot{\alpha}}=\lambda_{i}^{\alpha} \tilde{\lambda}_{i}^{\dot{\alpha}},
$$

we define the dual coordinates in the usual manner [25, 47],

$$
x_{i}^{\alpha \dot{\alpha}}-x_{i+1}^{\alpha \dot{\alpha}}=p_{i}^{\alpha \dot{\alpha}} .
$$

The dual $x_{i}$ define a light-like polygon in the dual space. On the other hand, a point in dual coordinate space corresponds to a (complex, projective) line in momentum twistor space. Two dual points are light-like separated if the corresponding lines in momentum twistor space intersect at some point in momentum twistor space. Thus the light-like polygon in dual space corresponds to a polygon in momentum twistor space with each line intersecting its two neighbouring lines as each dual point is light-like separated from its two neighbours. The $n$ momentum twistors associated to this configuration of $n$ light-like lines are defined via the incidence relations,

$$
Z_{i}^{A}=\left(\lambda_{i}^{\alpha}, \mu_{i}^{\dot{\alpha}}\right), \quad \mu_{i}^{\dot{\alpha}}=x_{i}^{\alpha \dot{\alpha}} \lambda_{i \alpha}=x_{i+1}^{\alpha \dot{\alpha}} \lambda_{i \alpha} .
$$

The momentum twistor transforms linearly under the action of dual conformal symmetry, as indicated by the fundamental $\operatorname{sl}(4)$ index $A$. Moreover the $n$ momentum twistors describing the polygon are free variables, in contrast to the dual points $x_{i}$ which obey the constraints of light-like separation from their neighbours. The dual point $x_{i}$ is associated to the line described by the pair $\left(Z_{i-1} Z_{i}\right)$ or $(i-1 i)$ for short. The incidence relations (2.3) allow one to express functions of the $x_{i}$ in terms of momentum twistors. For example we have

$$
x_{i j}^{2}=\frac{(i-1 i j-1 j)}{\langle i-1 i\rangle\langle j-1 j\rangle},
$$

where the four-brackets and two-brackets are defined as follows,

$$
(i j k l)=\epsilon_{A B C D} Z_{i}^{A} Z_{j}^{B} Z_{k}^{C} Z_{l}^{D}, \quad\langle i j\rangle=\lambda_{i}^{\alpha} \lambda_{j \alpha} .
$$



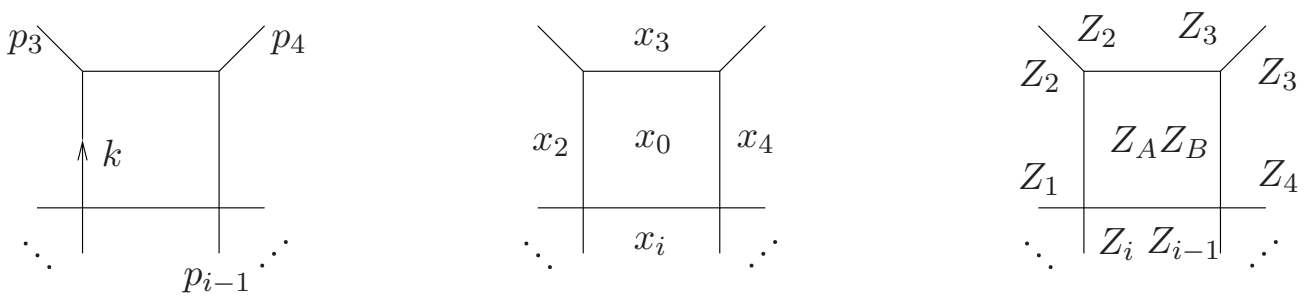

Figure 1. 'Two-mass-hard' integral in momentum space, dual space, and momentum twistor space variables, see equations (2.6) and (2.7).

The four-brackets are obviously dual conformal invariants while the two-brackets are invariant under just the Lorentz and (dual) translation transformations.

We can consider integrals over the space of lines in momentum twistor space $(A B)$. As we have discussed, this is equivalent to an integration over points in dual space. For example, the two-mass hard integral of figure 1 is given by

$$
\int \frac{d^{4} k}{i \pi^{2}} \frac{\left(p_{3}+\ldots+p_{i-1}\right)^{2}\left(p_{2}+p_{3}\right)^{2}}{k^{2}\left(k+p_{2}\right)^{2}\left(k+p_{2}+p_{3}\right)^{2}\left(k+p_{2}+\ldots+p_{i-1}\right)^{2}}=\int \frac{d^{4} x_{0}}{i \pi^{2}} \frac{x_{3 i}^{2} x_{24}^{2}}{x_{02}^{2} x_{03}^{2} x_{04}^{2} x_{0 i}^{2}},
$$

where the first expression is written in momentum space and the second one in dual coordinates. In twistor notation, this becomes

$$
\int \frac{d^{4} Z_{A B}}{i \pi^{2}} \frac{(23 i-1 i)(1234)}{(A B 12)(A B 23)(A B 34)(A B i-1 i)} .
$$

The integral (2.6) as written is infrared divergent, so its proper definition should involve a regulator. We will use the the AdS regularisation introduced in [10]. The latter allows us to stay in four dimensions and regulates the infrared divergences by masses that in turn are generated by a Higgs mechanism. For actual calculations we will use the regularisation where all masses are equal. In particular this means the outermost propagators in the planar loop integrals that we are studying are modified as follows,

$$
\frac{1}{x_{i j}^{2}} \longrightarrow \frac{1}{x_{i j}^{2}+m^{2}}
$$

In twistor space this has the effect that each of the propagator factors becomes

$$
\frac{1}{(A B i-1 i)} \longrightarrow \frac{1}{\langle A B i-1 i\rangle} \equiv \frac{1}{(A B i-1 i)+m^{2}\langle A B\rangle\langle i-1 i\rangle} .
$$

When doing this, there is a choice of adding $\mathcal{O}\left(\mathrm{m}^{2}\right)$ terms to the integrand. This is certainly relevant if one wishes to obtain the exact $m$-dependence of amplitudes on the Coulomb branch of $\mathcal{N}=4 \mathrm{SYM}$. In the cases considered here we are mostly interested in the case where $m$ is small. Unless an integral diverges linearly as $m \rightarrow 0$ one can then drop such numerator terms. This is the case for all integrals considered here.

Let us now discuss how such integrals appear in scattering amplitudes in $\mathcal{N}=4 \mathrm{SYM}$. At one loop, the MHV amplitudes are usually represented (or more precisely, their parityeven part) as a sum over so-called two-mass easy box integrals [48]. In [19], an alternative 

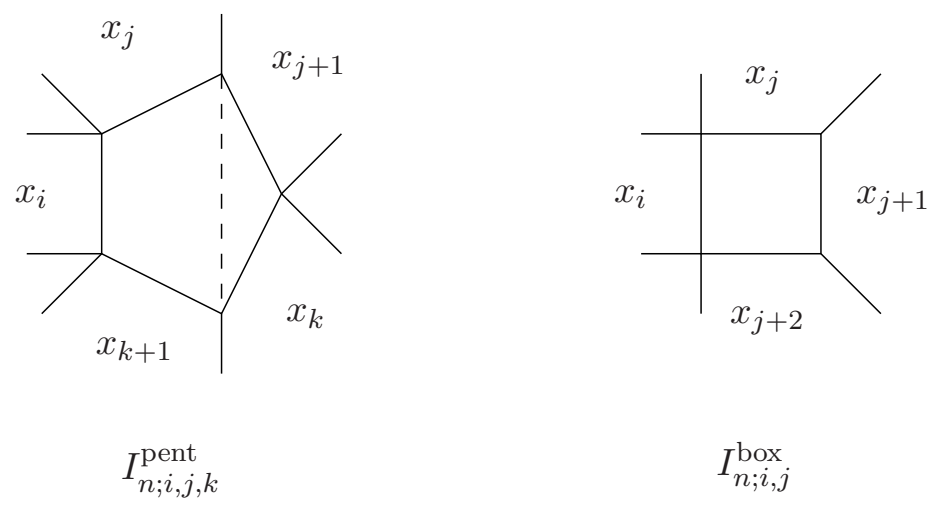

Figure 2. Integrals contributing to the one-loop MHV amplitude. The box integrand is obtained from the pentagon integrand by taking the soft limit $p_{n} \rightarrow 0$. The dashed line represents here the numerator which is denoted by wiggly line in [19].

form was given that uses pentagon integrals with certain twistor numerators. The formula given in [19] is a sum over the integrals shown in figure 2. Their integrand is given by

$$
I_{n ; i, j, k}^{\mathrm{pent}}=\frac{(i-1 i j k)(A B(j-1 j j+1) \cap(k-1 k k+1))}{(A B i-1 i)(A B j-1 j)(A B j j+1)(A B k-1 k)(A B k k+1)} .
$$

We use the notation $I$ for the integrand and $F$ for the function obtained after integration, ${ }^{1}$ i.e.

$$
F_{n ; i, j, k}^{\mathrm{pent}}=\int \frac{d^{4} Z_{A B}}{i \pi^{2}} I_{n ; i, j, k}^{\mathrm{pent}}
$$

where the number of points $n$ is implicit and appears only in the condition $n+1 \equiv 1$.

A comment is in order here regarding the numerator factors: In [19] both one-loop and two-loop amplitudes are written using numerators with wiggly lines representing

$$
(A B(i-1 i i+1) \cap(j-1 j j+1)):=(A i-1 i i+1)(B j-1 j j+1)-(B i-1 i i+1)(A j-1 j j+1) .
$$

The $\overline{\mathrm{MHV}}$ amplitude is obtained by changing wiggly lines to dashed lines that stand for $(A B i j)$, etc. In our discussion we do not distinguish between an integral and the parity conjugate integral because their difference is a parity odd integral which integrates to $\mathcal{O}\left(m^{2}\right)$. Therefore, in any diagram we can change all (but not just some) wiggly lines to dashed lines and vice-versa. In the following we will not distinguish between integrals with all dashed lines and those with all wiggly lines.

We now argue that all integrals appearing in the one-loop MHV amplitudes can be thought of as deriving from the pentagon integral. It is clear that pentagon integrals with lower number of legs can be obtained from a generic pentagon integral by taking soft limits. Therefore we only have to show how to obtain the box integrals of equation (2.7). As we will

\footnotetext{
${ }^{1}$ As was mentioned before, some of the integrals are in fact infrared divergent and require a regulator. In this section we mostly discuss properties of the (unregulated) loop integrand. It was argued in [19] that a correct expression for the loop integrand on the Coulomb branch of $\mathcal{N}=4 \mathrm{SYM}[10]$, up to $\mathcal{O}\left(\mathrm{m}^{2}\right)$ terms, can be obtained by adding masses to propagators on the perimeter of the diagams.
} 

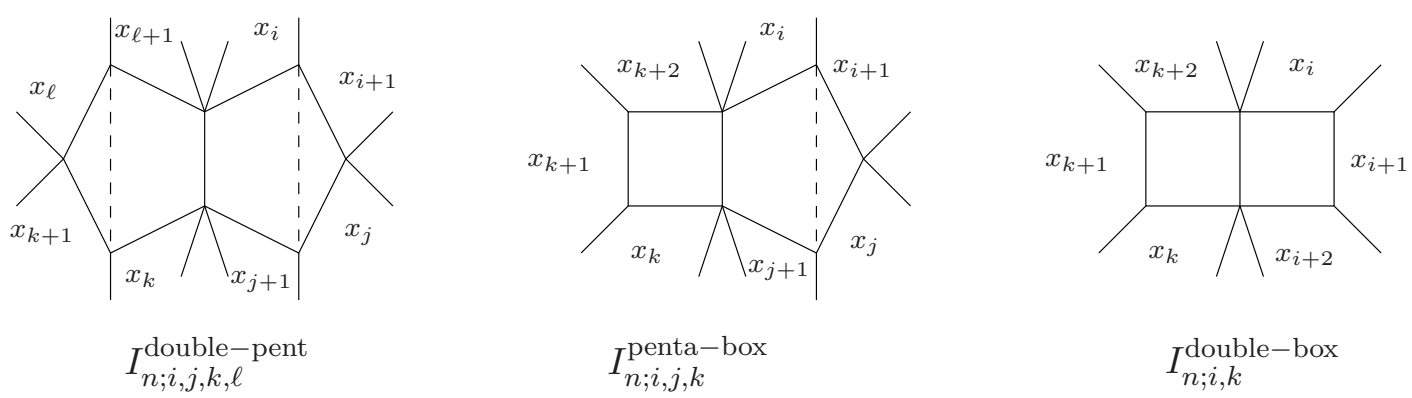

Figure 3. Integrals contributing to the two-loop MHV amplitude. The integrands of the double box and penta-box integrals are obtained from the double pentagon integrals by taking the soft limits. The dashed line have again the same meaning as in 1-loop case.

see presently, they are also obtained by taking soft limits. Indeed, consider the pentagon integral of figure 2 with $k=j+2$, so that only one external leg enters its rightmost corner. The soft limit of $p_{j+1} \rightarrow 0$ corresponds to letting

$$
Z_{j+1} \rightarrow \alpha Z_{j}+\beta Z_{j+2}
$$

Applying this limit to the integrand of the pentagon integral given in (2.10), we find

$$
\lim _{p_{j+1} \rightarrow 0} I_{n ; i, j, j+2}^{\text {pent }} \rightarrow I_{n-1 ; i, j}^{\text {box }},
$$

one reproduces exactly the integrand of the "two-mass hard" box. In this sense we can say that the one-loop MHV amplitude is built from a single master integral. We will show in section 3 that the latter satisfies a differential equation.

The situation is almost identical at the two-loop level. The expression given in [19] involves only the integrals shown in Fig 3. Recall that we denote the integrand of loop integrals by $I$ and the functions obtained after integration by $F$. For example, the first integral shown in figure 3 has the following definition,

$$
\begin{aligned}
I_{n ; i, j, k, \ell}^{\text {double-pent }}= & \frac{N}{(A B C D)} \frac{(C D(i-1 i i+1) \cap(j-1 j j+1))}{(C D i-1 i)(C D i i+1)(C D j-1 j)(C D j j+1)} \times \\
& \times \frac{(A B(k-1 k k+1) \cap(\ell-1 \ell \ell+1))}{(A B k-1 k)(A B k k+1)(A B \ell-1 \ell)(A B \ell \ell+1)},
\end{aligned}
$$

with the normalisation $N=(i j k \ell)$ and

$$
F_{n ; i, j, k, \ell}^{\text {double-pent }}=\int \frac{d^{4} Z_{A B}}{i \pi^{2}} \frac{d^{4} Z_{C D}}{i \pi^{2}} I_{n ; i, j, k, \ell}^{\text {double-pent }},
$$

where the number of points $n$ is implicit and appears only in the condition $n+1 \equiv 1$. The double pentagon integrals defined in (2.16) are in fact infrared finite [19]. Some of the pentagon and all of the box integrals we consider are infrared divergent and their definition is understood with the mass regulator in place, which leads to the modifications discussed above. From the one-loop case it is clear that the penta-box and double box integrals shown in figure 3 can be obtained from the double pentagon integral by taking subsequent soft limits. 


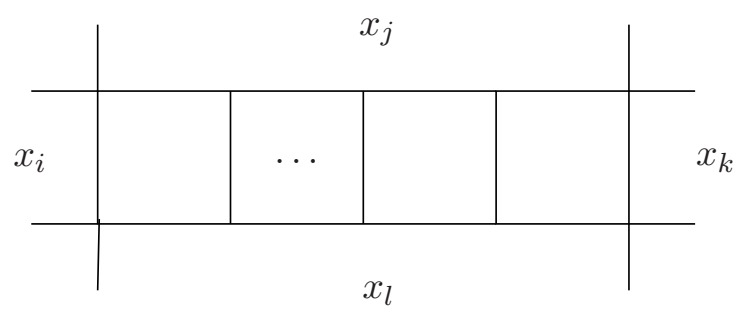

Figure 4. Ladder integrals defined in equations (3.1) and (3.11).

So, in summary, at each loop level the integrals appearing in the MHV amplitudes can be thought of as deriving from a single master topology. We will show in section 3 that the latter integral satisfies a second-order differential equation that reduces its loop order by one.

The relationship between the master integrals and the reduced integrals works at the level of the integrand. When the integration is taken into account, in some cases infrared divergences can lead to a non-commutativity of the soft limit and the regulator limit. We adopt the point of view that in those cases the relation to the master integral still implies a certain simplicity of the reduced integral. We show this explicitly for IR-divergent pentabox integrals in section 3, which satisfy the same type of differential equations.

Let us briefly discuss how many functionally different integrals of this class exist. The most general double pentagon integral of the type shown in figure 3 is the one where four of its external legs are doubled (when more legs are added, the function does not change). This is possible for the first time at $n=12$, e.g. $F_{12 ; 1,4,7,10}^{\text {double-pent }}$. In general, we can consider all integrals $F_{n ; i, j, k, \ell}^{\text {double-pent }}$ with zero, one, or two momenta flowing into these corners. Because of the symmetries of the pentagon, there are 18 different possibilities. However, because of the finiteness of the double pentagon integrals, all of them can be obtained from the most general case $F_{12 ; 1,4,7,10}^{\text {double-pent }}$ by taking soft limits. Finally, there are 12 penta-box integrals $F_{n ; i, j, k}^{\text {penta-box }}$ and 6 double box integrals of the type $F_{n ; i, k}^{\text {double-box }}$. The latter are all infrared divergent and therefore depend on the regulator $m^{2}$.

\section{Differential equations for loop integrals}

\subsection{Finite integrals}

\subsubsection{Ladders}

The ladders (or scalar boxes) provide a first example of a class of integrals which satisfy the type of differential equations we are interested in. To understand the differential equations for the ladder integrals it is convenient to use the dual coordinate notation. For the integrals appearing in the two-loop amplitude discussed in section 2 we will pass to the momentum twistor notation. We will begin with the one-loop box as an example, using dual variables to express the momenta,

$$
\tilde{F}^{(1)}\left(x_{i}, x_{j}, x_{k}, x_{l}\right)=\int \frac{d^{4} x_{r}}{i \pi^{2}} \frac{1}{x_{i r}^{2} x_{j r}^{2} x_{k r}^{2} x_{l r}^{2}} .
$$


Note that the points $x_{i}, x_{j}, x_{k}, x_{l}$ are generic points without any light-like separations. The $L$-loop version of this diagram is shown in figure 4. Since the integral (3.1) is covariant under conformal transformations of the $x$ coordinates, it can be expressed in terms of a function of the two available cross-ratios,

$$
u=\frac{x_{i j}^{2} x_{k l}^{2}}{x_{i k}^{2} x_{j l}^{2}}, \quad v=\frac{x_{i l}^{2} x_{j k}^{2}}{x_{i k}^{2} x_{j l}^{2}} .
$$

Thus we have

$$
\tilde{F}^{(1)}\left(x_{i}, x_{j}, x_{k}, x_{l}\right)=\frac{\Phi^{(1)}(u, v)}{x_{i k}^{2} x_{j l}^{2}},
$$

where the function $\Phi^{(1)}(u, v)$ is known [47, 49, 50]. In fact the function is best expressed in terms of the variables $z$ and $\bar{z}$ defined by ${ }^{2}$

$$
u=\frac{z \bar{z}}{(1-z)(1-\bar{z})}, \quad v=\frac{1}{(1-z)(1-\bar{z})} .
$$

Explicitly it is given by

$$
\Phi^{(1)}(u, v)=\frac{f^{(1)}(z, \bar{z})}{z-\bar{z}}, \quad f^{(1)}(z, \bar{z})=\log (z \bar{z})\left(\operatorname{Li}_{1}(z)-\operatorname{Li}_{1}(\bar{z})\right)-2\left(\operatorname{Li}_{2}(z)-\operatorname{Li}_{2}(\bar{z})\right) .
$$

The integral $\tilde{F}^{(1)}$ satisfies a simple second order differential equation. The reason for this is that acting with the Laplace operator on one of the external points, $x_{i}$ say, produces a delta function under the integral [25],

$$
\square_{i} \frac{1}{x_{i r}^{2}}=-4 i \pi^{2} \delta^{(4)}\left(x_{i}-x_{r}\right)
$$

This has the effect of localising the integral completely, giving a simple secondorder equation,

$$
\square_{i} \tilde{F}^{(1)}\left(x_{i}, x_{j}, x_{k}, x_{l}\right)=\frac{-4}{x_{i j}^{2} x_{i k}^{2} x_{i l}^{2}} .
$$

On the other hand [25], acting on the form of $\tilde{F}^{(1)}$ given in (3.3) one obtains

$$
\square_{i} \tilde{F}^{(1)}\left(x_{i}, x_{j}, x_{k}, x_{l}\right)=\frac{x_{j k}^{2} x_{k l}^{2}}{x_{i k}^{6} x_{j l}^{4}} \Delta_{u, v} \Phi^{(1)}(u, v)
$$

where $\Delta_{u, v}$ is a second-order differential operator,

$$
\Delta_{u, v}=u \partial_{u}^{2}+v \partial_{v}^{2}+(u+v-1) \partial_{u} \partial_{v}+2 \partial_{u}+2 \partial_{v} .
$$

The equality of the two expressions (3.7) and (3.8) is a second order differential equation for the function $\Phi^{(1)}$ or equivalently $f^{(1)}$. Expressing the equation in terms of $z$ and $\bar{z}$ it reads,

$$
z \partial_{z} \bar{z} \partial_{\bar{z}} f^{(1)}(z, \bar{z})=\frac{z}{z-1}-\frac{\bar{z}}{\bar{z}-1} .
$$

\footnotetext{
${ }^{2}$ Note that $z$ and $\bar{z}$ are real and independent in Minkowski signature whereas they are complex conjugate to each other in Euclidean signature.
} 
The main points we wish to stress are that the action of the operator removes the loop integration, leaving a rational function behind. The existence of a simple equation also means that the underlying function is a relatively simple pure transcendental function of degree two.

The one-loop integral we have been discussing is just the first in an infinite sequence of ladder integrals. These integrals (along with a large class of equivalent integrals [25]) exhibit an iterative structure,

$$
\tilde{F}^{(L)}\left(x_{i}, x_{j}, x_{k}, x_{l}\right)=\int \frac{d^{4} x_{r}}{i \pi^{2}} \frac{x_{j l}^{2}}{x_{i r}^{2} x_{j r}^{2} x_{l r}^{2}} \tilde{F}^{(L-1)}\left(x_{r}, x_{j}, x_{k}, x_{l}\right) .
$$

As before the integrals are invariant under conformal transformations of the $x_{i}$ and so are given in terms of functions of the the two cross-ratios,

$$
\tilde{F}^{(L)}\left(x_{i}, x_{j}, x_{k}, x_{l}\right)=\frac{\Phi^{(L)}(u, v)}{x_{i k}^{2} x_{j l}^{2}} .
$$

As before the function $\Phi^{(n)}$ is best expressed in terms of the variables $z$ and $\bar{z}$,

$$
\Phi^{(L)}(u, v)=\frac{f^{(L)}(z, \bar{z})}{z-\bar{z}},
$$

where

$$
f^{(L)}(z, \bar{z})=\sum_{r=0}^{L} \frac{(-1)^{r+L}(2 L-r) !}{r !(L-r) ! L !} \log ^{r}(z \bar{z})\left(\operatorname{Li}_{2 L-r}(z)-\operatorname{Li}_{2 L-r}(\bar{z})\right) .
$$

The fact that the one-loop box satisfies a differential equation guarantees that all the ladder integrals do. One can see that Laplace operator $\square_{i}$ is effectively acting only on a one-loop box subintegral which we have already seen reduces to a rational function. Thus the operation reduces the loop order of the ladder integral,

$$
\square_{i} \tilde{F}^{(L)}\left(x_{i}, x_{j}, x_{k}, x_{l}\right)=-4 \frac{x_{j l}^{2}}{x_{i j}^{2} x_{i l}^{2}} \tilde{F}^{(L-1)}\left(x_{i}, x_{j}, x_{k}, x_{l}\right) .
$$

The functions appearing in the explicit expressions exhibit corresponding differential equations

$$
z \partial_{z} \bar{z} \partial_{\bar{z}} f^{(L)}(z, \bar{z})=f^{(L-1)}(z, \bar{z})
$$

\subsubsection{Pentaladders}

The integrals involving twistor numerators can also satisfy differential equations. We will begin with integrals which are similar to the ladders of the previous section. We would like to consider the finite pentaladder integrals, beginning with one of the one-loop pentagon integrals discussed in section 2. We will write in dual coordinate notation to begin with and later move to the momentum twistor notation. The integral we would like to consider is the seven-point one-loop pentagon integral, which is shown for arbitrary number of loops in figure $5(\mathrm{a})$,

$$
\tilde{F}_{\mathrm{pl}}^{(1)}\left(x_{1}, x_{3}, x_{4}, x_{5}, x_{6}\right)=\int \frac{d^{4} x_{r}}{i \pi^{2}} \frac{x_{a r}^{2}}{x_{1 r}^{2} x_{3 r}^{2} x_{4 r}^{2} x_{5 r}^{2} x_{6 r}^{2}} .
$$




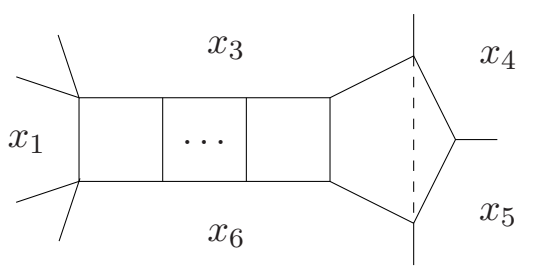

(a)

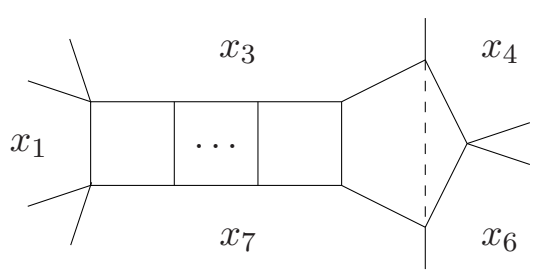

(b)

Figure 5. figure (a) represents the seven-point integrals defined in (3.17) and (3.24), and (b) shows the eight-point integrals defined in (3.28) and (3.32).

Note that we have not normalised the integral so (3.17) is dimensionful. The point $x_{a}$ is the magic complex point which is null-separated from every point on the null lines $\left(x_{3}, x_{4}\right),\left(x_{4}, x_{5}\right)$ and $\left(x_{5}, x_{6}\right)$. It is one of the solutions to

$$
x_{a 3}^{2}=x_{a 4}^{2}=x_{a 5}^{2}=x_{a 6}^{2}=0 .
$$

The other solution is its parity conjugate.

Just like the ladder integrals, the pentagon we are considering is conformally covariant so we can write it in the following way

$$
\tilde{F}_{\mathrm{pl}}^{(1)}\left(x_{1}, x_{3}, x_{4}, x_{5}, x_{6}\right)=\frac{x_{1 a}^{2}}{x_{14}^{2} x_{15}^{2} x_{36}^{2}} \frac{\Psi^{(1)}(u, v)}{(1-u-v)},
$$

where $u$ and $v$ are the two non-vanishing conformal cross-ratios,

$$
u=\frac{x_{13}^{2} x_{46}^{2}}{x_{14}^{2} x_{36}^{2}}, \quad v=\frac{x_{16}^{2} x_{35}^{2}}{x_{15}^{2} x_{36}^{2}} .
$$

We have chosen to make a factor of $1 /(1-u-v)$ explicit on the r.h.s. of (3.19) for later convenience.

In terms of the quantities introduced in section 2 we have

$$
x_{14}^{2} x_{15}^{2} x_{36}^{2} \tilde{F}_{\mathrm{pl}}^{(1)}\left(x_{1}, x_{3}, x_{4}, x_{5}, x_{6}\right)=\Psi^{(1)}(u, v)=F_{7 ; 1,3,5}^{\text {pent }}
$$

The integral satisfies a differential equation very similar to the equation for the ladder integrals,

$$
\square_{1} \tilde{F}_{\mathrm{pl}}^{(1)}=-4 \frac{x_{1 a}^{2}}{x_{13}^{2} x_{14}^{2} x_{15}^{2} x_{16}^{2}} .
$$

This equation translates into a second order p.d.e. for the function $\Psi^{(1)}$,

$$
u v \partial_{u} \partial_{v} \Psi^{(1)}(u, v)=1
$$

Just as for the ladders we can define multi-loop pentaladder integrals via an iterative structure. For example we can consider the integrals, see figure 5(a),

$$
\tilde{F}_{\mathrm{pl}}^{(L)}\left(x_{1}, x_{3}, x_{4}, x_{5}, x_{6}\right)=\int \frac{d^{4} x_{r}}{i \pi^{2}} \frac{x_{36}^{2}}{x_{1 r}^{2} x_{3 r}^{2} x_{6 r}^{2}} \tilde{F}_{\mathrm{pl}}^{(L-1)}\left(x_{r}, x_{3}, x_{4}, x_{5}, x_{6}\right) .
$$


Equivalently we can write them using the ladder integrals,

$$
\tilde{F}_{\mathrm{pl}}^{(L)}\left(x_{1}, x_{3}, x_{4}, x_{5}, x_{6}\right)=\int \frac{d^{4} x_{r}}{i \pi^{2}} \frac{x_{a r}^{2} x_{36}^{2}}{x_{3 r}^{2} x_{4 r}^{2} x_{5 r}^{2} x_{6 r}^{2}} \tilde{F}^{(L-1)}\left(x_{1}, x_{3}, x_{r}, x_{6}\right) .
$$

As before, we will use conformal symmetry in the $x$ variables to write the integral in the form

$$
\tilde{F}_{\mathrm{pl}}^{(L)}\left(x_{1}, x_{3}, x_{4}, x_{5}, x_{6}\right)=\frac{x_{1 a}^{2}}{x_{14}^{2} x_{15}^{2} x_{36}^{2}} \frac{\Psi^{(L)}(u, v)}{(1-u-v)} .
$$

Now applying the Laplace operator gives the following differential equation for $\Psi^{(L)}$,

$$
(1-u-v) u v \partial_{u} \partial_{v} \Psi^{(L)}(u, v)=\Psi^{(L-1)}(u, v),
$$

where we define $\Psi^{(0)}(u, v) \equiv 1-u-v$.

We can also consider pentaladder integrals with a massive corner on the pentagon subintegral, see figure 5(b). At one loop the integral takes the form

$$
\tilde{F}_{\mathrm{pl}}^{(1)}\left(x_{1}, x_{3}, x_{4}, x_{6}, x_{7}\right)=\int \frac{d^{4} x_{r}}{i \pi^{2}} \frac{x_{a r}^{2}}{x_{1 r}^{2} x_{3 r}^{2} x_{4 r}^{2} x_{6 r}^{2} x_{7 r}^{2}} .
$$

Here the point $x_{a}$ is light-like separated from $x_{3}, x_{4}, x_{6}, x_{7}$. It is convenient to write this integral as

$$
\tilde{F}_{\mathrm{pl}}^{(1)}\left(x_{1}, x_{3}, x_{4}, x_{6}, x_{7}\right)=\frac{x_{1 a}^{2}}{x_{14}^{2} x_{16}^{2} x_{37}^{2}} \frac{\tilde{\Psi}^{(1)}(u, v, w)}{(1-u-v+u v w)},
$$

where the three cross-ratios are

$$
u=\frac{x_{13}^{2} x_{47}^{2}}{x_{14}^{2} x_{37}^{2}}, \quad v=\frac{x_{17}^{2} x_{36}^{2}}{x_{16}^{2} x_{37}^{2}}, \quad w=\frac{x_{37}^{2} x_{46}^{2}}{x_{36}^{2} x_{47}^{2}} .
$$

We remark that the function $\Psi^{(1)}(u, v)$ discussed before can be obtained from $\tilde{\Psi}^{(1)}(u, v, w)$ by taking the (smooth) soft limit $w \rightarrow 0$.

Following the same logic as before we obtain the equation

$$
u \partial_{u} v \partial_{v} \tilde{\Psi}^{(1)}(u, v, w)=1 .
$$

A similar analysis holds for the $L$-loop case defined by

$$
\tilde{F}_{\mathrm{pl}}^{(L)}\left(x_{1}, x_{3}, x_{4}, x_{6}, x_{7}\right)=\int \frac{d^{4} x_{r}}{i \pi^{2}} \frac{x_{a r}^{2} x_{37}^{2}}{x_{3 r}^{2} x_{4 r}^{2} x_{6 r}^{2} x_{7 r}^{2}} \tilde{F}^{(L-1)}\left(x_{1}, x_{3}, x_{r}, x_{7}\right) .
$$

Writing the integral as a function of the cross-ratios,

$$
\tilde{F}_{\mathrm{pl}}^{(L)}\left(x_{1}, x_{3}, x_{4}, x_{6}, x_{7}\right)=\frac{x_{1 a}^{2}}{x_{14}^{2} x_{16}^{2} x_{37}^{2}} \frac{\tilde{\Psi}^{(L)}(u, v, w)}{(1-u-v+u v w)},
$$

we find the differential equation,

$$
(1-u-v+u v w) u v \partial_{u} \partial_{v} \tilde{\Psi}^{(L)}(u, v, w)=\Psi^{(L-1)}(u, v, w),
$$

with $\tilde{\Psi}^{(0)}(u, v, w) \equiv 1-u-v+u v w$. 


\subsubsection{Pentaladders reloaded}

In the case of the pentaladder integrals we can also arrive at the differential equation by considering the momentum twistor representation of the integrals. A clue to constructing the right operator comes from the fact that the Laplacian naively annihilates the integrand,

$$
\square_{1} \frac{1}{x_{1 r}^{2}}=0 \quad \text { (naive) } .
$$

Of course we have seen that this does not imply that the integral itself is annihilated by the Laplace operator because there is an anomaly in the form of the delta function as in (3.6).

Let us recall that if one writes the one-loop finite pentagon integral using momentum twistors it takes the form,

$$
\begin{aligned}
\Psi^{(1)}(u, v) & =F_{7 ; 1,3,5}^{\mathrm{pent}}=\int \frac{d^{4} Z_{A B}}{i \pi^{2}} I_{7 ; 1,3,5}^{\mathrm{pent}}, \\
I_{7 ; 1,3,5}^{\mathrm{pent}} & =\frac{(456[7)(1] 234)(A B 35)}{(A B 71)(A B 23)(A B 34)(A B 45)(A B 56)} .
\end{aligned}
$$

It is possible to construct an operator acting on the momentum twistor variables which also annihilates the integrand $I_{7 ; 1,3,5}^{\text {pent }}$. Let us first introduce some notation to deal with twistor derivatives.

We define a twistor derivative $O_{i j}$ as

$$
O_{i j}=Z_{i} \cdot \frac{\partial}{\partial Z_{j}}
$$

It acts trivially on four-brackets, $O_{i j}(j \ell k m)=(i \ell k m)$. The normalized integrals are homogenous in all external twistors, which can be also written as

$$
O_{i i} I=0, \quad i=1, \ldots n .
$$

Let us do a trivial exercise which will be important in the following discussion. Let us start with the rational function of four-bracket, $I=(A B 24) /(A B 23)(A B 34)$ and act on it with the operator $O_{12}$. We immediately get

$$
O_{12} I=\frac{(A B 14)}{(A B 23)(A B 34)}-\frac{(A B 24)(A B 13)}{(A B 23)^{2}(A B 34)}=\frac{(A B 12)}{(A B 23)^{2}}
$$

where we used Shouten identity $(A B 14)(A B 23)-(A B 24)(A B 13)=(A B 12)(A B 34)$. Now, we see that the dependence of twistor $Z_{3}$ is just through the four-bracket $(A B 23)$. If we now act with the operator $O_{23}$, then we get zero, therefore

$$
\mathrm{O}_{23} O_{12} \frac{(A B 24)}{(A B 23)(A B 34)}=0 \text {. }
$$

Most of the equations we will derive later are based on the same principle.

In the case of the pentagon integral the analysis above leads us to define the following operator

$$
\tilde{O}_{234}=N_{\mathrm{pl}} O_{34} O_{23} N_{\mathrm{pl}}^{-1}, \quad N_{\mathrm{pl}}=(456[7)(1] 234) .
$$


The operator $\tilde{O}_{234}$ naively annihilates the integrand $I_{n ; 1,3,5}^{\text {pent }}$,

$$
\tilde{O}_{234} I_{7 ; 1,3,5}^{\text {pent }}=0 \quad \text { (naive) } .
$$

We have written 'naive' here because, just as for the Laplace operator, we must remember that we are going to perform an integration and there may be anomalies like the delta function left behind. In fact we can easily convince ourselves that there are indeed anomalous terms in place of the naive zero on the r.h.s. of (3.43). Let us express the action of the operator on the integral function $\Psi^{(1)}(u, v)$. We recall that the cross-ratios take the following form in terms of momentum twistors,

$$
u=\frac{(7123)(3456)}{(7134)(2356)}, \quad v=\frac{(7156)(2345)}{(7145)(2356)} .
$$

Acting with the operator $\tilde{O}$ on the integral function we find

$$
\tilde{O}_{234} \Psi^{(1)}(u, v)=-\frac{(7135) N_{\mathrm{pl}}}{(7145)(7134)(3456)} u \partial_{u} v \partial_{v} \Psi^{(1)}(u, v) .
$$

We already know from acting with the Laplace operator that the r.h.s. of (3.45) is not zero. Indeed the second-order derivative of $\Psi^{(1)}$ is 1 according to (3.23). Writing the equation without the factors of $N_{\mathrm{pl}}$ on both sides leads us to the following relation,

$$
O_{34} O_{23} \int \frac{d^{4} Z_{A B}}{i \pi^{2}} \frac{(A B 35)}{(A B 71)(A B 23)(A B 34)(A B 45)(A B 56)}=-\frac{(7135)}{(7134)(7145)(3456)} .
$$

This equation can then be used whenever we find the one-loop pentagon as a subintegral. In particular one can derive the differential equations for the multi-loop pentaladder integrals. As an example we discuss here the finite two-loop pentabox integral,

$$
\begin{aligned}
& \Psi^{(2)}(u, v)= \\
& \quad \int \frac{d^{4} Z_{A B}}{i \pi^{2}} \frac{d^{4} Z_{C D}}{i \pi^{2}} \frac{(A B 35) N_{\mathrm{pl}}(2356)}{(A B 23)(A B 34)(A B 45)(A B 56)(A B C D)(C D 56)(C D 71)(C D 23)} .
\end{aligned}
$$

Applying the operator $\tilde{O}_{234}$ and using the identity (3.46) we find

$$
\tilde{O}_{234} \Psi^{(2)}(u, v)=-\frac{(2356)}{(3456)} \int \frac{d^{4} Z_{C D}}{i \pi^{2}} \frac{N_{\mathrm{pl}}(C D 35)}{(C D 71)(C D 23)(C D 34)(C D 45)(C D 56)} .
$$

Using (3.45) on the l.h.s. and the definition of the one-loop pentagon (3.36), (3.37) on the r.h.s. we arrive at

$$
\frac{(7135) N_{\mathrm{pl}}}{(7134)(7145)(2356)} u \partial_{u} v \partial_{v} \Psi^{(2)}(u, v)=\Psi^{(1)}(u, v) .
$$

By using cyclic identities one finds that the four-brackets on the l.h.s. can be written in terms of $u$ and $v$ and we arrive at the equation

$$
(1-u-v) u \partial_{u} v \partial_{v} \Psi^{(2)}(u, v)=\Psi^{(1)}(u, v),
$$


exactly as derived from the Laplace operator in (3.27) in the case $L=2$. We have rederived the equation for the two-loop finite pentaladder to simplify the equations but the derivation for $L$ loops is essentially identical and leads to (3.27) for general $L$.

In summary, we have derived the second-order equations (3.27) by acting with the twistor differential operator $\mathrm{O}_{34} \mathrm{O}_{23}$ on the pentagon sub-integral, thanks to equation (3.46). What this implies is that whenever we have an integral with the pentagon integral as a sub-integral, we can use this mechanism and generate a second-order equation by using (3.46). Importantly, this is also possible in cases where one cannot apply the Laplace operator, as e.g. for the double pentagon integrals that we will discuss in section 3.1.4. Before doing this, we are going to discuss the generalisation where the pentagon sub-integral has a massive corner.

In the case of the pentaladders with a massive corner one again finds certain operators which annihilate the integrand. Let us consider the integrand of the one-loop pentagon with three massive legs,

$$
I_{8 ; 1,3,6}^{\mathrm{pent}}=\frac{N_{\mathrm{pm}}(A B 36)}{(A B 81)(A B 23)(A B 34)(A B 56)(A B 67)},
$$

with $N_{\mathrm{pm}}=(567[8)(1] 234)$. The integrand is annihilated by certain twistor operators,

$$
N_{\mathrm{pm}} O_{24} O_{42} N_{\mathrm{pm}}^{-1} I_{8 ; 1,3,6}^{\mathrm{pent}}=0, \quad N_{\mathrm{pm}} O_{75} O_{57} N_{\mathrm{pm}}^{-1} I_{8 ; 1,3,6}^{\mathrm{pent}}=0 .
$$

Applying these operators to the explicit function we actually find that they annihilate it, i.e. there is no anomaly associated to this operator on $F_{8 ; 1,3,6}^{\text {pent }}$. In fact we will see that $F_{8 ; 1,3,6}^{\text {pent }}$ is given explicitly by $[51]^{3}$

$$
\begin{aligned}
F_{8 ; 1,3,6}^{\text {pent }} \equiv \tilde{\Psi}^{(1)}(u, v, w)= & \log u \log v+\operatorname{Li}_{2}(1-u)+\operatorname{Li}_{2}(1-v)+\operatorname{Li}_{2}(1-w) \\
& -\operatorname{Li}_{2}(1-u w)-\operatorname{Li}_{2}(1-v w)
\end{aligned}
$$

where the cross-ratios take the form

$$
u=\frac{(8123)(3467)}{(8134)(2367)}, \quad v=\frac{(8167)(2356)}{(8156)(2367)}, \quad w=\frac{(2367)(3456)}{(2356)(3467)} .
$$

We remark that the function $\Psi^{(1)}(u, v)$ describing the seven-point integral $F_{7 ; 1,3,5}^{\text {pent }}$ can be obtained from $\tilde{\Psi}^{(1)}(u, v, w)$ by taking a (smooth) soft limit,

$$
\lim _{w \rightarrow 0} \tilde{\Psi}^{(1)}(u, v, w)=\Psi^{(1)}(u, v) .
$$

Using (3.53) we then find that the following homogeneous equations are satisfied,

$$
N_{\mathrm{pm}} O_{24} O_{42} N_{\mathrm{pm}}^{-1} F_{8 ; 1,3,6}^{\mathrm{pent}}=0, \quad N_{\mathrm{pm}} O_{75} O_{57} N_{\mathrm{pm}}^{-1} F_{8 ; 1,3,6}^{\mathrm{pent}}=0 .
$$

Writing these in terms of the cross-ratios we find,

$$
\begin{aligned}
& {\left[(1-u)^{2} \partial_{u} u \partial_{u}+(1-w)^{2} \partial_{w} w \partial_{w}-u v(1-u)(1-w) \partial_{u} \partial_{v}\right.} \\
& \left.\quad+(1-u)(1-w)(1+u w) \partial_{u} \partial_{w}-v(1-w)^{2} \partial_{v} \partial_{w}\right] \tilde{\Psi}^{(1)}(u, v, w)=0
\end{aligned}
$$

\footnotetext{
${ }^{3}$ Here we are assuming that all dual distances are spacelike. One must carefully analytically continue to the regions where some of them become timelike.
} 
and the equation obtained from swapping $u$ and $v$. As before, we can generalise these equations to higher-loop integrals. Note, however, that e.g. the first operator given in (3.56) , $\mathrm{O}_{24} \mathrm{O}_{42}$, contains a derivative acting on $Z_{2}$. We can only use (3.56) on a pentagon subintegral of a higher-loop integral if the latter does not depend on $Z_{2}$ elsewhere, to avoid cross terms. This is the case if there are at least two additional legs attached at the intersection of the pentagon integral with the rest of the diagram (both additional legs must be on the same side of the diagram).

We will now present a further mechanism for generating differential equations that will yield an equation that is valid without the above restriction. To begin with, let us apply the differential operator $O_{24} O_{75}$ to the integrand $I_{8 ; 1,3,6}^{\text {pent }}$. A short calculation shows that

$$
O_{24} O_{75} I_{8 ; 1,3,6}^{\mathrm{pent}}=N_{\mathrm{pm}} \frac{(A B 36)}{(A B 81)(A B 34)^{2}(A B 56)^{2}}
$$

i.e. we have cancelled two propagators. Notice that for obtaining this expression no cyclic identities were needed. What this means is that the calculation would go through unchanged in the presence of a $+m^{2}$ regularisation, so that we do not expect contact terms here. Also note that the resulting integral is finite thanks to the numerator factor. Since it is also dual conformal, it can only have a very restricted variable dependence. In fact, one can easily convince oneself that up to a trivial factor, this integral is a dual conformal three-point function that can depend on the dual points $x_{1}, x_{4}, x_{6}$ only. Since there are no cross-ratios at three points, it must be a rational function. Let us compute the latter. Introducing Feynman parameters, we find

$$
\int d \alpha_{i} \frac{\alpha_{1} \alpha_{4} \alpha_{6}(3618)}{\left[\alpha_{1} \alpha_{4}(8134)+\alpha_{1} \alpha_{6}(8156)+\alpha_{4} \alpha_{6}(3456)\right]^{3}}
$$

The remaining projective integrals are easily carried out, with the result being proportional to

$$
\frac{(3618)}{(8134)(8156)(3456)}
$$

Since the differential operator commutes with the normalisation of the integrand we find

$$
O_{24} O_{75} F_{8 ; 1,3,6}^{\mathrm{pent}} \propto N_{\mathrm{pm}} \frac{(3681)}{(8134)(8156)(3456)}
$$

One can easily check this equation on the explicit function (3.53).

The equation for the sub-integral can immediately be applied to higher-loop integrals containing it. There is no subtlety since the twistor derivatives only act on $Z_{4}$ and $Z_{5}$, and the latter variables are separated from the rest of the integral.

We can write down several other operators which follow the same pattern. The idea is simple: if we can manage to reduce the number of propagators to three, dual conformal 


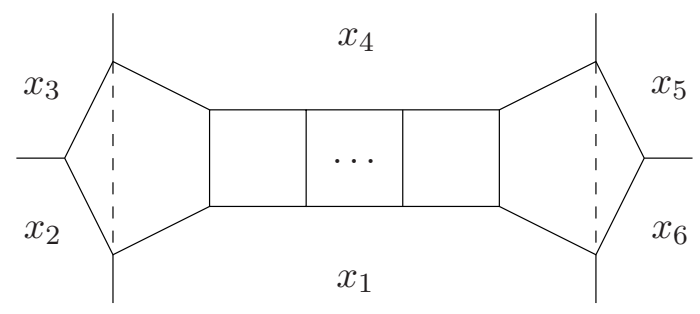

Figure 6. Six-point double pentaladder integral (3.62).

symmetry will imply that the resulting integral is rational. ${ }^{4}$ An example is given by $O_{42} O_{75}$. Note, however, that in that case the corresponding equation can only be used iteratively in cases where there is no $Z_{2}$ dependence in the remainder of the integral.

In conclusion, whenever we encounter a pentagon sub-integral of the type considered in this section, either with or without massive corner, we can always find at least one differential equation that can be used iteratively. In some cases we can even find further equations. In particular what this means is that we can find differential equations for all double pentagon integrals appearing in the MHV amplitudes. In the next section we are going to spell these equations out explicitly for the case of an infinite class of double pentaladder integrals.

\subsubsection{Double pentaladders}

The fact that we have shown that one can derive differential equations using the momentum twistors is important because it allows us to find equations in cases where no Laplace operator derivation exists. As an example we will consider the six-point finite double pentaladder integrals. These integrals can be defined in terms of the pentaladders of the previous subsection,

$$
\tilde{F}_{\mathrm{dp}}^{(L)}\left(x_{1}, x_{2}, x_{3}, x_{4}, x_{5}, x_{6}\right)=\int \frac{d^{4} x_{r} x_{a r}^{2} x_{14}^{2}}{x_{1 r}^{2} x_{2 r}^{2} x_{3 r}^{2} x_{4 r}^{2}} \tilde{F}_{\mathrm{pl}}^{(L-1)}\left(x_{r}, x_{4}, x_{5}, x_{6}, x_{1}\right),
$$

see figure 6 . Conformal symmetry in the $x$ variables tells us that we can write

$$
\tilde{F}_{\mathrm{dp}}^{(L)}\left(x_{1}, x_{2}, x_{3}, x_{4}, x_{5}, x_{6}\right)=\frac{x_{a b}^{2}}{x_{26}^{2} x_{35}^{2} x_{14}^{2}} \Omega^{(L)}(u, v, w),
$$

where $u, v$ and $w$ are the three cross-ratios,

$$
u=\frac{x_{13}^{2} x_{46}^{2}}{x_{14}^{2} x_{36}^{2}}, \quad v=\frac{x_{24}^{2} x_{15}^{2}}{x_{14}^{2} x_{25}^{2}}, \quad w=\frac{x_{35}^{2} x_{26}^{2}}{x_{25}^{2} x_{36}^{2}} .
$$

\footnotetext{
${ }^{4}$ Naively one might find it surprising that dual conformal symmetry is helping us here, since the momentum twistors already made that symmetry manifest. Indeed with six twistor variables it is possible to form homogeneous cross-ratios but the final result contains no extra functional dependence on these variables. The reason is that we are combining the symmetry with the fact that our functions are Feynman integrals built from propagators, which leads to additional restrictions on the possible dependence on the twistor variables.
} 
To derive differential equations for these integrals we need to express them in momentum twistor notation. We will consider the finite six-point two-loop double pentagon integral as an example,

$$
\begin{aligned}
& \Omega^{(2)}(u, v, w)=F_{6 ; 1,3,4,6}^{\text {double-pent }} \\
& \quad=\int \frac{d^{4} Z_{A B}}{i \pi^{2}} \frac{d^{4} Z_{C D}}{i \pi^{2}} \frac{(A B 13)(C D 46) N_{\mathrm{dp}}}{(A B 61)(A B 12)(A B 23)(A B 34)(A B C D)(C D 45)(C D 56)(C D 61)},
\end{aligned}
$$

where $N_{\mathrm{dp}}=(2345)(5612)(3461)$. In terms of the momentum twistors the three cross-ratios take the form,

$$
u=\frac{(6123)(3456)}{(6134)(2356)}, \quad v=\frac{(1234)(4561)}{(1245)(6134)}, \quad w=\frac{(2345)(1256)}{(2356)(1245)} .
$$

We see that the double pentagon integral contains the one-loop pentagon that we studied in the previous subsection as a subintegral. Thus we can apply the same kind of differential operator and derive a differential equation. The operator we wish to apply here is

$$
\tilde{O}_{612}=N_{\mathrm{dp}} O_{12} O_{61} N_{\mathrm{dp}}^{-1} \text {. }
$$

Acting with this operator on the the double pentagon integral and using the relation (3.46) with the appropriate relabelling we find

$$
\begin{aligned}
\tilde{O}_{612} \Omega^{(2)}(u, v, w) & =-\frac{1}{(1234)} \int \frac{d^{4} Z_{C D}}{i \pi^{2}} \frac{N_{\mathrm{dp}}(C D 13)(C D 46)}{(C D 12)(C D 23)(C D 34)(C D 45)(C D 56)(C D 61)} \\
& =-\frac{(3461)}{(1234)} \Omega^{(1)}(u, v, w) .
\end{aligned}
$$

Here $\Omega^{(1)}(u, v, w)$ is the one-loop finite hexagon integral,

$$
\Omega^{(1)}(u, v, w)=\int \frac{d^{4} Z_{C D}}{i \pi^{2}} \frac{N_{\mathrm{hex}}(C D 13)(C D 46)}{(C D 12)(C D 23)(C D 34)(C D 45)(C D 56)(C D 61)},
$$

where $N_{\text {hex }}=(2345)(5612)$.

From the discussion in section 3.1.3 it is clear that the above equations can be straightforwardly generalised to the case where the pentagon sub-integrals have a massive middle leg.

\subsection{Divergent integrals}

Some of the integrals appearing in the MHV amplitudes are infrared divergent. These divergences are regulated by masses as explained in section 2 . In this section we show that one can find similar differential equations as above, that hold up to $\mathcal{O}\left(\mathrm{m}^{2}\right)$.

\subsubsection{One-loop box integrals}

As explained in section 2, they are special cases of the general pentagon topology with magic numerator, when no external legs are attached to the rightmost corner. In this case, one obtains a two-mass hard box integral, whose integrand is given by

$$
F_{n ; i, 2}^{\text {box }}=\int \frac{d^{4} x_{0}}{i \pi^{2}} \frac{x_{24}^{2} x_{3 i}^{2}}{\left(x_{20}^{2}+m^{2}\right)\left(x_{30}^{2}+m^{2}\right)\left(x_{40}^{2}+m^{2}\right)\left(x_{i 0}^{2}+m^{2}\right)},
$$


Unlike the generic pentagon integral $F_{n ; i, j, k}^{\text {pent }}$ the two-mass hard integral is infrared divergent and depend explicitly on the mass $m$ that regulates the infrared divergences, see section 2 . Here we discuss that the differential equations we found for the finite integrals work in exactly the same way here, up to $\mathcal{O}\left(\mathrm{m}^{2}\right)$ corrections. To understand why this is the case, consider again the Laplace operator, but acting on a massive propagator. ${ }^{5}$ We have

$$
\square_{1} \frac{1}{x_{1 i}^{2}+m^{2}}=-8 \frac{m^{2}}{\left(x_{1 i}^{2}+m^{2}\right)^{3}}=-4 i \pi^{2} \delta^{(4)}\left(x_{1 i}^{\mu}\right)+\mathcal{O}\left(m^{2}\right),
$$

so that in this case we would indeed "undo" one integration as in the massless case, but up to $\mathcal{O}\left(m^{2}\right)$ corrections. As we discuss presently, this leads second-order differential equations for this integral, at least for $n \geq 5$. We will first discuss the generic case where (3.70) represents indeed a two-mass hard integral, and subsequently discuss the degenerate onemass case where $i=4$ or $i=n$.

In the former case, there is one off-shell leg that we can apply the Laplace operator to, see equation (3.71). This leads to a differential equation valid to $\mathcal{O}\left(\mathrm{m}^{2}\right)$, namely

$$
x_{2 i}^{2} x_{3 i}^{2} x_{4 i}^{2} \square_{i}\left(x_{24}^{2} x_{3 i}^{2}\right)^{-1} F_{n ; i ; 2}^{\text {box }}=-4+\mathcal{O}\left(m^{2}\right) .
$$

We can explicitly verify (3.72) by differentiating the known answer for the two-mass hard integral. In terms of Feynman parameters, it is given by

$$
F_{n ; i ; 2}^{\mathrm{box}}=\int d \alpha_{i} \frac{x_{24}^{2} x_{3 i}^{2} \delta\left(\alpha_{2}+\alpha_{3}+\alpha_{4}+\alpha_{i}-1\right)}{\left[\alpha_{2} \alpha_{4} x_{24}^{2}+\alpha_{i}\left(\alpha_{2} x_{2 i, m}^{2}+\alpha_{3} x_{3 i, m}^{2}+\alpha_{4} x_{4 i, m}^{2}\right)+m^{2}\right]^{2}},
$$

where $x_{2 i, m}^{2}:=x_{2 i}^{2}+m^{2}$, etc. One obtains

$$
F_{n ; i ; 2}^{\mathrm{box}}=\frac{1}{2} \log ^{2}\left(y_{1} y_{2} y_{3}\right)+2 \operatorname{Li}_{2}\left(1-y_{2}\right)+2 \operatorname{Li}_{2}\left(1-y_{3}\right)+\mathcal{O}\left(m^{2}\right)
$$

where we used the variables $y_{1}=x_{24}^{2} / m^{2}, y_{2}=x_{3 i}^{2} / x_{2 i}^{2}, y_{3}=x_{3 i}^{2} / x_{4 i}^{2}$. Just like in section 3.1, we can also find twistor derivatives that lead to differential equations. However, the two-mass hard integral is infrared divergent, and here we have dropped certain $\mathcal{O}\left(\mathrm{m}^{2}\right)$ terms. When applying differential operators to an infrared divergent subintegral, e.g. in the double box case, one must be very careful when making such approximations. We postpone a detailed analysis of the double box integrals to future work.

\subsubsection{Penta-box integrals}

A class of finite pentaladder integrals was already discussed in section 3.1. Here we show that the IR divergent penta-box integrals also satisfy analogous differential equations, up to $\mathcal{O}\left(m^{2}\right)$.

\footnotetext{
${ }^{5}$ In this case one could also note that massive propagators are formally identical to free AdS bulk-toboundary propagators, and use the corresponding equation of motion, see [52]. For this it is necessary to consider situation with generic masses $m_{i}$, but this is no restriction thanks to dual conformal symmetry. This would yield equations that are exact in the $m_{i}$, i.e. that do not rely on taking the masses small w.r.t. the Mandelstam invariants. It is an interesting open question how to extend this efficiently to the on-shell case.
} 

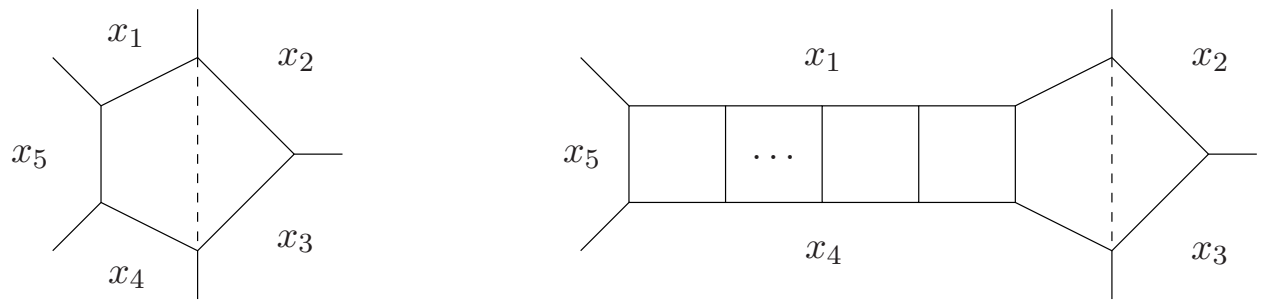

Figure 7. Five-point penta-ladder integrals considered in the text.

Let us consider the pentaladder integrals shown in figure 7 . The discussion for other divergent pentaladder integrals would be very similar. At one loop, we have

$$
F_{5 ; 5,1,3}^{\mathrm{pent}}=\int \frac{d^{4} Z_{A B}}{i \pi^{2}} \frac{(2345)(4512)(A B 13)}{(51 A B)(12 A B)(23 A B)(34 A B)(45 A B)},
$$

Since all external legs are on-shell, we cannot use the Laplace operator. We already know from section 3.1.3 that in such cases twistor derivatives can have the same effect. Indeed, consider the action of the differential operator

$$
N O_{32} O_{43} N^{-1}=Z_{3} \cdot \frac{\partial}{\partial Z_{2}} Z_{4} \cdot \frac{\partial}{\partial Z_{3}}+\frac{(5134)}{(1245)} Z_{4} \cdot \frac{\partial}{\partial Z_{3}},
$$

where $N=(2345)(4512)$, One can check that it annihilates its integrand, up to $\mathcal{O}\left(m^{2}\right)$ terms. As discussed above, one can think of the mass as regulating distributional terms that arise when acting with the derivatives. Let us now find out what happens when we act with the operator in (3.76) on the function obtained after integration. Computing the one-loop integral $F_{5 ; 5,1,3}^{\text {pent }}$ directly using Feynman parameters in the small $m^{2}$ limit, we have (see appendix B)

$$
F_{5 ; 5,1,3}^{\text {pent }}=-\frac{1}{2} \log ^{2}\left(y_{1} y_{2} y_{3}\right)-2 \operatorname{Li}_{2}\left(1-y_{1}\right)-2 \operatorname{Li}_{2}\left(1-y_{2}\right)+\frac{\pi^{2}}{6}+\mathcal{O}\left(m^{2}\right) .
$$

Here $y_{1}=x_{35}^{2} / x_{13}^{2}, y_{2}=x_{25}^{2} / x_{24}^{2}, y_{3}=x_{14}^{2} / m^{2}$, and we recall the relation of the dual variables to the momentum twistors,

$$
x_{13}^{2}=\frac{(5123)}{\langle 51\rangle\langle 23\rangle}, x_{24}^{2}=\frac{(1234)}{\langle 12\rangle\langle 34\rangle}, x_{35}^{2}=\frac{(2345)}{\langle 23\rangle\langle 45\rangle}, x_{14}^{2}=\frac{(3451)}{\langle 34\rangle\langle 51\rangle}, x_{25}^{2}=\frac{(4512)}{\langle 45\rangle\langle 12\rangle} .
$$

Using the chain rule and acting on (3.77) one can easily verify that

$$
\frac{(5123)}{(4513)}\left[Z_{3} \cdot \frac{\partial}{\partial Z_{2}} Z_{4} \cdot \frac{\partial}{\partial Z_{3}}+\frac{(5134)}{(1245)} Z_{4} \cdot \frac{\partial}{\partial Z_{3}}\right] F_{5 ; 5,1,3}^{\text {pent }}=-1+\mathcal{O}\left(m^{2}\right),
$$

i.e. the r.h.s. is not zero but a simple rational term. In other words, it is still true that the differential operator "undoes" the integration, at least up to $\mathcal{O}\left(\mathrm{m}^{2}\right)$.

We now discuss the all-loop generalisation of these equations. The two-loop version of $F_{5 ; 5,1,3}^{\text {pent }}$ is

$$
F_{5 ; 5,1,3}^{\text {penta-box }}=\int \frac{d^{4} Z_{A B} d^{4} Z_{C D}\left(i \pi^{2}\right)^{-2} N(C D 13)}{(A B 34)(A B 45)(A B 51)(A B C D)(C D 51)(C D 12)(C D 23)(C D 34)},
$$


where $N=(3451)(2345)(4512)$.

As in the previous sections, we need to discuss the pentagon subintegral. In fact the subintegral is the same finite integral that was discussed in section 3.1, with the difference that since the two-loop integral is IR divergent its propagators contain $+m^{2}$ pieces. However, we already know that the special numerator makes the subintegral finite, and therefore the effect of the masses in the pentagon propagator should be only $\mathcal{O}\left(\mathrm{m}^{2}\right)$. ${ }^{6}$ Therefore we can neglect these masses when acting with the differential operator, and it is clear that we will obtain differential equations completely analogous to those discussed in section 3.1. The only difference is that by construction they are only valid up to $\mathcal{O}\left(\mathrm{m}^{2}\right)$ corrections. Therefore we have

$$
\frac{(5123)}{(4513)} \mathrm{NO}_{32} \mathrm{O}_{43} N^{-1} F_{5 ; 5,1,3}^{\text {penta-box }}=F_{5 ; 5,1,3}^{\text {pent }}+\mathcal{O}\left(m^{2}\right) .
$$

Of course one can immediately generalise this equation to arbitrary loop order, by replacing the integral on the l.h.s. and r.h.s. by the $L$-loop and $(L-1)$-loop penta-box integrals, respectively. Finally, the other divergent penta-box integrals which differ in the arrangement of external legs all satisfy analogous differential equations.

In summary, we have found differential equations for all integrals contributing to the one- and two-loop MHV amplitudes in $\mathcal{N}=4 \mathrm{SYM}$, except for the six double box integrals. The differential equations we find are iterative in the loop order and apply to infinite series of on-shell loop integrals. We expect that integrals contributing to higher-loop MHV amplitudes and other helicity configurations are constrained in a similar way.

The same mechanisms for generating differential equations that we described above should also hold for the double box integrals. However a separate analysis is required there since they do not contain IR finite subintegrals. We leave this for future work. (An encouraging fact is that in appendix $\mathrm{A}$ we find differential operators that naively annihilate the double box integrand.) Of course, the double box integrals are in some sense the simplest integrals appearing in the two-loop MHV amplitudes, so perhaps they could also be computed by other means. Also, they are related to the more general integrals that do satisfy differential equations through soft limits. If one can control the non-commutativity of the soft limit with the regulator limit this can also provide a useful tool for computing them.

\section{Solving the differential equations}

\subsection{Harmonic polylogarithms}

We will now discuss solving the differential equations. The solutions we find are typically written in terms of polylogarimic functions. Here we will give a brief introduction to the notation we use which can be found in $[53,54]$.

The simple harmonic polylogarithm functions are given in terms of iterated integrals. We start from the functions

$$
H(0 ; x)=\log x, \quad H(1 ; x)=-\log (1-x) .
$$

\footnotetext{
${ }^{6}$ This argument presupposes that the divergent box subintegral produces only logarithmic but no linear divergences in $m^{2}$. This is the case for all integrals we consider.
} 
Their derivatives are given by the fractions

$$
\frac{d}{d x} H(a ; x)=f(a ; x), \quad f(0 ; x)=\frac{1}{x}, \quad f(1 ; x)=\frac{1}{1-x} .
$$

We can now define weight $w$ harmonic polylogarithms depending on a vector with entries of ones and zeros. For a vector of all zeros of length $w$ (written $\overrightarrow{0}_{w}$ ) we have

$$
H\left(\overrightarrow{0}_{w} ; x\right)=\frac{1}{w !} \log ^{w} x .
$$

Further harmonic polylogarithms depending on a more general vector $\vec{m}_{w}=\left(a, \vec{m}_{w-1}\right)$ can be defined by repeated integration,

$$
H\left(\vec{m}_{w} ; x\right)=\int_{0}^{x} d x^{\prime} f\left(a ; x^{\prime}\right) H\left(\vec{m}_{w-1} ; x^{\prime}\right) .
$$

Below we save space by writing the vector $\vec{m}_{w}$ as a subscript. Finally we can use the shorthand notation where proceeding from right to left a zero is eliminated if it is to the left of a non-zero entry while at the same time one is added to the value of that entry. For example

$$
H_{3,2}(x)=H(0,0,1,0,1 ; x) .
$$

There also exist harmonic polylogarithms with negative arguments but we will not need them here.

The simple harmonic polylogarithms defined above are all single variable functions. However they can be generalised to functions of more than one variable. In general the relevant functions are the Goncharov polylogarithms. Here we will only need functions of two variables at most which can be described in terms of two-dimensional harmonic polylogarithms. These functions are defined in much the same way as the simple harmonic polylogarithms but we allow slightly more general arguments of the vector $\vec{m}_{w}$. This is done by enlarging the set of weight one functions and fractions to include

$$
H(1-y ; x)=-\log \left(1-\frac{x}{1-y}\right), \quad f(1-y ; x)=\frac{1}{1-x-y} .
$$

\subsection{Solution for seven-point pentagon and penta-box integral}

Let us consider the equation for $\Psi^{(1)}$, the one-loop pentagon integral. The equation is

$$
u v \partial_{u} \partial_{v} \Psi^{(1)}(u, v)=1 .
$$

The general solution is

$$
\Psi^{(1)}(u, v)=\log u \log v+g(u)+g(v)
$$

for some single variable function $g$.

Let us recall that we chose to write the pentaladder integrals is the following way,

$$
\tilde{F}_{\mathrm{pl}}^{(L)}\left(x_{1}, x_{3}, x_{4}, x_{5}, x_{6}\right)=\frac{x_{1 a}^{2}}{x_{14}^{2} x_{15}^{2} x_{36}^{2}} \frac{\Psi^{(L)}(u, v)}{(1-u-v)} .
$$


This form makes it clear that the function $\Psi^{(L)}(u, v)$ should have a simple zero when $1-u-v=0$ since the integral clearly has no special singular behaviour on this region. Thus we have

$$
\Psi^{(L)}(u, 1-u)=0 .
$$

The boundary condition (4.10) implies that the function $g$ should satisfy

$$
g(u)+g(1-u)=-\log u \log (1-u) .
$$

The solution of (4.11) is ambiguous up to any function odd in swapping $u$ and $1-u$. Thus we find

$$
g(u)=\operatorname{Li}_{2}(1-u)-\frac{1}{2} \zeta(2)+h(u)-h(1-u),
$$

for any single variable function $h$.

We need some extra data to fix the function $h(u)-h(1-u)$. If we adopt maximal transcendentality (which translates here to the fact that $h(u)$ can only be a sum of harmonic polylogarithms) and also the requirement that there are no branch points of $\Psi^{(1)}(u, v)$ for $u>0, v>0$ then we fix $h(u)=0$. Indeed if we assume that we can write $h(u)$ as a sum of harmonic polylogarithms of degree 2 ,

$$
h(u)=a \operatorname{Li}_{2}(u)+b \log ^{2} u+c \log u \operatorname{Li}_{1}(u)+d \operatorname{Li}_{1}^{2}(u),
$$

then the only solution where $h(u)-h(1-u)$ has no branch points at $u=1$ is indeed $h=0$. Thus we deduce that

$$
\Psi^{(1)}(u, v)=\log u \log v+\operatorname{Li}_{2}(1-u)+\operatorname{Li}_{2}(1-v)-\zeta(2) .
$$

Given the correct answer for $\Psi^{(1)}$ we can go on to determine the next pentaladder function $\Psi^{(2)}$. The equation we have to solve is

$$
(1-u-v) u v \partial_{u} \partial_{v} \Psi^{(2)}(u, v)=\Psi^{(1)}(u, v) .
$$

It is helpful to divide by the factor $(1-u-v) u v$ and use partial fractions to write

$$
\partial_{u} \partial_{v} \Psi^{(2)}(u, v)=\Psi^{(1)}(u, v)\left[\frac{1}{u v}+\frac{1}{u(1-u-v)}+\frac{1}{v(1-u-v)}\right] .
$$

Now we look for particular solutions which reproduce each of the three terms on the r.h.s., i.e.

$$
\begin{aligned}
\partial_{u} \partial_{v} H^{0}(u, v) & =\frac{\Psi^{(1)}(u, v)}{u v}, \\
\partial_{u} \partial_{v} H^{u}(u, v) & =\frac{\Psi^{(1)}(u, v)}{u(1-u-v)}, \\
\partial_{u} \partial_{v} H^{v}(u, v) & =\frac{\Psi^{(1)}(u, v)}{v(1-u-v)} .
\end{aligned}
$$


We find the following solution for $H^{0}$,

$$
\begin{aligned}
H^{0}(u, v)= & 2 \zeta(4)+\frac{1}{2} \zeta(2)^{2}-\zeta(2)\left(\operatorname{Li}_{2}(1-u)+\operatorname{Li}_{2}(1-v)\right) \\
& +\log u \log v\left(\frac{1}{4} \log u \log v+\operatorname{Li}_{2}(1-u)+\operatorname{Li}_{2}(1-v)-\zeta(2)\right) \\
& +\log u\left(2 H_{2,1}(1-v)-\operatorname{Li}_{3}(1-u)+\zeta(3)\right) \\
& +\log v\left(2 H_{2,1}(1-u)-\operatorname{Li}_{3}(1-v)+\zeta(3)\right) \\
& -3\left(H_{3,1}(1-u)+H_{3,1}(1-v)\right)
\end{aligned}
$$

where we have used the notation of harmonic polylogarithms [53]. In fact $H_{2,1}$ is a Nielsen polylogarithm and can be reexpressed in terms of ordinary trilogarithms but we prefer not to do so here. For $H^{u}$ we find, ${ }^{7}$

$$
\begin{aligned}
H^{u}(u, v)= & \zeta(2)\left(H_{0,1-v}(u)-\operatorname{Li}_{2}(u)-\log u \log (1-v)\right)+H_{0,0}(u) H_{1,0}(v) \\
& +\log v\left(H_{0,1-v, 0}(u)-H_{2,0}(u)\right)+\log u H_{1,1,0}(v) \\
& -H_{2,1,0}(u)+H_{0,1-v, 1,0}(u)+H_{0,1-v}(u) H_{1,0}(v),
\end{aligned}
$$

where in addition to harmonic polylogarithms we have used the two-dimensional harmonic polylogarithms of [54]. We have neglected some obvious simplifications such as $H_{0,0}(u)=$ $\frac{1}{2} \log ^{2} u$ and $H_{1,0}(u)=-\operatorname{Li}_{2}(u)-\log u \log (1-u)$. By symmetry we have also

$$
H^{v}(u, v)=H^{u}(v, u)
$$

Thus the general solution to the differential equation (4.15) is

$$
\Psi^{(2)}(u, v)=H^{0}(u, v)+H^{u}(u, v)+H^{v}(u, v)+g(u)+g(v),
$$

for some single variable function $g$. The function $g$ can be constrained by the fact that $\Psi^{(2)}(u, v)$ should vanish when $1-u-v=0$. Again assuming that it is purely transcendental and has no spurious branch cuts (although as presented, the branch cut structure of $H^{u}$ is not obvious) fixes the function. We find

$$
\begin{aligned}
g(u)= & -\frac{1}{2} \zeta(2)^{2}-3 \zeta(4)+\zeta(2)\left(\frac{1}{2} \log ^{2} u+\operatorname{Li}_{2}(1-u)\right) \\
& +\log u\left(\operatorname{Li}_{3}(1-u)+H_{2,1}(1-u)-3 \zeta(3)\right)+2 H_{3,1}(1-u)+3 H_{2,1,1}(1-u) .
\end{aligned}
$$

We have checked that this combination of functions numerically matches the Mellin-Barnes representation of the integral in the triangular region $u>0, v>0, u+v<1$ to a high degree of accuracy. Thus we are confident that (4.23) together with (4.20), (4.21), (4.22), and (4.24) correctly give the two-loop pentaladder function.

\footnotetext{
${ }^{7}$ We thank L. Dixon for pointing out a typo in a previous version of this formula.
} 


\subsection{Equations for eight-point pentagon and pentaladder integrals}

We would now like to discuss the equations for integrals with pentagon subintegrals with massive corners. For example we have the pentaladder integrals with a massive corner,

$$
\tilde{F}_{\mathrm{pl}}^{(L)}\left(x_{1}, x_{3}, x_{4}, x_{6}, x_{7}\right)=\frac{x_{1 a}^{2}}{x_{14}^{2} x_{16}^{2} x_{37}^{2}} \frac{\tilde{\Psi}^{(L)}(u, v, w)}{(1-u-v+u v w)},
$$

Note that the way we have chosen to write the r.h.s. means that $\tilde{\Psi}^{(L)}(u, v, w)$ vanishes when $1-u-v+u v w=0$ because the integral has no pole in this region.

As we have seen for the pentaladders with a massive corner we have two sources of differential equations. Firstly from applying the Laplace operator we have:

$$
(1-u-v+u v w) u v \partial_{u} \partial_{v} \tilde{\Psi}^{(L)}(u, v, w)=\Psi^{(L-1)}(u, v, w) .
$$

Then in the twistor language we can apply the operator

$$
\mathrm{O}_{24} \mathrm{O}_{75}
$$

which also produces a rational function. In fact we have

$$
N_{\mathrm{pm}} O_{24} O_{75} N_{\mathrm{pm}}^{-1} \tilde{\Psi}^{(1)}(u, v, w)=O_{24} O_{75} \tilde{\Psi}^{(1)}(u, v, w)=\frac{N_{\mathrm{pm}}(3681)}{(3456)(3481)(5681)} .
$$

This leads to the equation

$$
\begin{gathered}
w\left[(1-w) \partial_{w}\left((1-w) \partial_{w}+v(1-v) \partial_{v}+u(1-u) \partial_{u}\right)+u(1-u) v(1-v) \partial_{u} \partial_{v}\right] \tilde{\Psi}^{(1)}(u, v, w) \\
=1-u-v+u v w \equiv \tilde{\Psi}^{(0)}(u, v, w) .
\end{gathered}
$$

More generally we have for the $L$-loop pentaladder with a massive corner,

$$
\begin{gathered}
w\left[(1-w) \partial_{w}\left((1-w) \partial_{w}+v(1-v) \partial_{v}+u(1-u) \partial_{u}\right)+u(1-u) v(1-v) \partial_{u} \partial_{v}\right] \tilde{\Psi}^{(L)}(u, v, w) \\
=\tilde{\Psi}^{(L-1)}(u, v, w) .
\end{gathered}
$$

From (4.26) we know the result of acting with the final term in the operator on the l.h.s. so we can rewrite the equation in a simpler way. We find

$$
w \partial_{w}\left[(1-w) \partial_{w}+u(1-u) \partial_{u}+v(1-v) \partial_{v}\right] \tilde{\Psi}^{(1)}(u, v, w)=1-u-v
$$

or more generally for $L$ loops,

$$
w \partial_{w}\left[(1-w) \partial_{w}+u(1-u) \partial_{u}+v(1-v) \partial_{v}\right] \tilde{\Psi}^{(L)}(u, v, w)=\frac{(1-u-v) \tilde{\Psi}^{(L-1)}(u, v, w)}{1-u-v+u v w} .
$$

Now let us consider (4.26) restricted to $w=1$. We find that the operator factorises,

$$
u(1-u) \partial_{u} v(1-v) \partial_{v} \tilde{\Psi}^{(L)}(u, v, 1)=\tilde{\Psi}^{(L-1)}(u, v, 1) .
$$

In the one-loop case we then have simply

$$
u \partial_{u} v \partial_{v} \tilde{\Psi}^{(1)}(u, v, 1)=1 .
$$


and so

$$
\tilde{\Psi}^{(1)}(u, v, 1)=\log u \log v+f(u)+f(v),
$$

for some single variable function $f$. We recall that $\tilde{\Psi}^{(L)}(u, v, w)$ vanishes when $1-u-v+$ $u v w=0$. In our case where $w=1$ this implies that $\tilde{\Psi}^{(1)}(u, v, 1)$ vanishes when $u=1$ or $v=1$. Thus we have

$$
f(u)+f(1)=0,
$$

which implies that $f(u)=0$ and so

$$
\tilde{\Psi}^{(1)}(u, v, 1)=\log u \log v .
$$

In the two-loop case it is again simple,

$$
u(1-u) \partial_{u} v(1-v) \partial_{v} \tilde{\Psi}^{(2)}(u, v, 1)=\log u \log v .
$$

Integrating we find

$$
\tilde{\Psi}^{(2)}(u, v, 1)=\left[H_{0,0}(u)+H_{1,0}(u)\right]\left[H_{0,0}(v)+H_{1,0}(v)\right]+f(u)+f(v)
$$

for some single variable function $f$. As before we have the condition that $\tilde{\Psi}^{(L)}(u, v, 1)$ vanishes when $u=1$ or $v=1$. Thus we find

$$
-\zeta(2)\left[H_{0,0}(u)+H_{1,0}(u)\right]+f(u)+f(1)=0 .
$$

Setting $u=1$ this tells us

$$
f(1)=-\frac{1}{2} \zeta(2)^{2}
$$

Thus we have

$$
f(u)=\zeta(2)\left[H_{0,0}(u)+H_{1,0}(u)+\frac{1}{2} \zeta(2)\right]
$$

and we have solved for $\tilde{\Psi}^{(2)}(u, v, 1)$ quite simply.

Now we can return to the other equation (4.32). Let us perform the $w$ integral,

$$
\left[(1-w) \partial_{w}+u(1-u) \partial_{u}+v(1-v) \partial_{v}\right] \tilde{\Psi}^{(L)}(u, v, w)=X^{(L)}(u, v, w)+f_{X}^{(L)}(u, v)
$$

where

$$
X^{(L)}(u, v, w)=(1-u-v) \int_{1}^{w} \frac{d t \tilde{\Psi}^{(L-1)}(u, v, t)}{t(1-u-v+u v t)}
$$

and $f_{X}(u, v)$ is an arbitrary two-variable function. We can determine $f_{X}$ however by setting set $w=1$ in (4.43) and we find

$$
\left[u(1-u) \partial_{u}+v(1-v) \partial_{v}\right] \tilde{\Psi}^{(L)}(u, v, 1)=f_{X}^{(L)}(u, v) .
$$

The 1.h.s. is known from the Laplace operator as shown above so given $\tilde{\Psi}^{(L)}(u, v, 1)$ we know $f_{X}^{(L)}(u, v)$. In the one-loop case we have

$$
f_{X}^{(1)}(u, v)=(1-u) \log v+(1-v) \log u
$$


while

$$
X^{(1)}(u, v, w)=(1-u-v) \int_{1}^{w} \frac{d t}{t}=(1-u-v) \log w .
$$

Thus (4.43) becomes a first order equation for $\tilde{\Psi}^{(1)}(u, v, w)$. One can easily convince oneself that the unique solution is (3.53) given the known boundary condition for $\tilde{\Psi}^{(1)}(u, v, 1)$ or equivalently $\tilde{\Psi}^{(1)}(u, v, 0)=\Psi^{(1)}(u, v)$.

More generally we always have a first order equation for $\tilde{\Psi}^{(L)}(u, v, w)$ and thus one can write the function in a simple integral form.

\subsection{Equations for the six-point hexagon and double-pentagon integrals}

Let us now discuss the simplest cases of the double pentaladder integrals. To start we will consider the finite six-point double-pentagon, defined in (3.65). In terms of the twistor variables the independent cross-ratios take the form

$$
u=\frac{(6123)(3456)}{(6134)(2356)}, \quad v=\frac{(1234)(4561)}{(1245)(6134)}, \quad w=\frac{(2345)(1256)}{(2356)(1245)} .
$$

Translating the equation (3.68) into an equation for the function $\Omega^{(2)}$ we find

$$
\begin{aligned}
& {\left[\left(1-u-v+\frac{\tilde{\epsilon}}{2}\right) u v \partial_{u} \partial_{v}+\frac{\tilde{\epsilon}-2 u}{2} v w \partial_{v} \partial_{w}\right.} \\
& \left.\quad+\frac{\tilde{\epsilon}-2 v}{2} u w \partial_{u} \partial_{w}-\frac{\tilde{\epsilon} w}{2} \partial_{w}(1-w) \partial_{w}\right] \Omega^{(2)}(u, v, w)=\Omega^{(1)}(u, v, w) .
\end{aligned}
$$

with $\tilde{\epsilon}=\sqrt{\delta^{2}-4 u v w}+\delta$ and $\delta=u+v+w-1$.

In fact the sign of the square root is ambiguous and so the equation separates into two second order equations, one homogeneous, the other inhomogeneous,

$$
\begin{aligned}
{\left[(1-u-v) u v \partial_{u} \partial_{v}-u v w \partial_{v} \partial_{w}-u v w \partial_{u} \partial_{w}\right] \Omega^{(2)}(u, v, w) } & =\Omega^{(1)}(u, v, w), \\
{\left[u v \partial_{u} \partial_{v}+u w \partial_{u} \partial_{w}+v w \partial_{v} \partial_{w}-w \partial_{w}(1-w) \partial_{w}\right] \Omega^{(2)}(u, v, w) } & =0 .
\end{aligned}
$$

In fact we can set $w=0$ in (4.50) and we find

$$
(1-u-v) u v \partial_{u} \partial_{v} \Omega^{(2)}(u, v, 0)=\Omega^{(1)}(u, v, 0) .
$$

This equation is identical in form to that for the functions $\Psi^{(L)}(u, v)$ from the pentaladder with a massless corner. At one loop we have in fact

$$
\Omega^{(1)}(u, v, w)=\log u \log v+\operatorname{Li}_{2}(1-u)+\operatorname{Li}_{2}(1-v)+\operatorname{Li}_{2}(1-w)-2 \zeta(2),
$$

and indeed $\Omega^{(1)}(u, v, 0)=\Psi^{(1)}(u, v)$. We have verified numerically from the Mellin-Barnes representation that the same holds at two loops, $\Omega^{(2)}(u, v, 0)=\Psi^{(2)}(u, v)$, so this twovariable slice of the function $\Omega^{(2)}$ is the same function that we have solved for from the pentaladder equation.

Combining the two equations (4.50), (4.51) we can write a factorised operator,

$$
w \partial_{w}\left[-u(1-u) \partial_{u}-v(1-v) \partial_{v}+(1-u-v)(1-w) \partial_{w}\right] \Omega^{(2)}(u, v, w)=\Omega^{(1)}(u, v, w) .
$$

After performing one integration on $w$ we have a first-order equation for the function $\Omega^{(2)}$. 


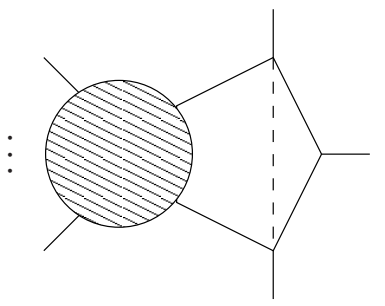

Figure 8. The iterative differential equations can always be obtained if a key sub-integral is present, as shown in the figure. The salient feature is the presence of a "magic numerator", as indicated by the dashed line. The number of differential equations that can be obtained for an integral depends on the precise configuration of external legs.

\section{Conclusion and outlook}

In this paper we derived a new type of differential equations for on-shell integrals. The equations are second order and reduce the loop order by one. We presented several classes of infinite of integrals closed under the differential equations. In particular, these include most integrals appearing in $\mathcal{N}=4 \mathrm{MHV}$ amplitudes to two loops. One may speculate that the same should be true to all loop orders.

We also discussed how such differential equations can be solved by making reasonable assumptions about the boundary conditions. The explicit solution for a finite two-loop seven-point and a divergent two-loop five-point integral was given. The answers are relatively simple and one can hope that one can solve for infinite series of such integrals, as is possible for the off-shell ladder integrals [47, 49, 50]. This would be an important step toward summing the perturbation series in $\mathcal{N}=4$ SYM. See [55] for a study of the summation of the off-shell latter integrals.

The main motivation of this study was to contribute to the determination of the allorder S-matrix of $\mathcal{N}=4 \mathrm{SYM}$. One may ask whether there is a short-cut by deriving differential equations for the whole amplitude, as opposed to individual integrals. In appendix A we found differential operators that annihilate (naively) the integrand of the one-loop MHV amplitude. In order to understand these differential operators better it is important to systematically investigate distributional terms that can appear and lead to inhomogeneous terms in differential equations. A related open question is the link of our differential equations to the conformal symmetry of $\mathcal{N}=4 \mathrm{SYM}$.

We expect our differential equations to be useful for other theories as well. Due to their nice properties it is certainly desirable to include the integrals with magic numerators introduced in [19] and discussed in [43] and the present paper in the integral basis. The necessary numerator identities were given in [43]. Moreover, one can find differential equations of the type presented here for larger classes of integrals. This is clear from the way the differential equations were derived: all one needs is a certain sub-topology with "magic" numerators. In the cases considered in this paper the latter appeared on top of a pentagon topology, see figure 8 , which made sure that the integrals are dual conformal. However, one could drop this requirement and extend the current method to integrals that are not dual conformal. 
Another natural extension of our work is to use different regulators. Some of the integrals discussed here are finite, and in the case of infrared divergences we used a mass regulator, allowing us to stay in four dimensions, which is very natural when working with twistor variables. However, this is not mandatory. Although the details may be slightly different, the mechanism for finding differential equations should work with other regulators as well. E.g. in dimensional regularisation one might expect similar equations to hold, at least to some order in $\epsilon$, where the dimension is $4-2 \epsilon$.

It is interesting to obtain equations exact in $m^{2}$, which would allow to discuss e.g. scattering amplitudes on the Coulomb branch of $\mathcal{N}=4$ SYM. Equations exact in $m^{2}$ could also be useful to study integrals appearing in realistic amplitudes involving massive particles, see e.g. [56]. It should be possible to derive such equations at least in some cases, perhaps by noticing that four-dimensional massive propagators are formally identical to AdS bulk-to-boundary propagators [52].

\section{Acknowledgments}

It is a pleasure to thank N. Arkani-Hamed, J. Bourjaily, F. Cachazo and A. Kotikov for useful discussions. J.M.D. and J.M.H. are indebted to N. Arkani-Hamed for several invitations to the IAS, where part of this work was done.

\section{A Twistor differential operators}

In section 3, we showed that all integrals appearing in the one- and two-loop MHV amplitudes with at least five external legs satisfy differential equations. In order to obtain these equations it was crucial to find differential operators that can act on a sub-integral and reduce it to a rational function.

In order to find the relevant twistor differential operators we found it useful to systematically write down all differential operators that have of property of (naively) annihilating the integrand of a given integral. Of course this is not equivalent to finding a useful differential equation for the function after integration, but it served as a helpful starting point for our above analysis. Here we present more details of the mechanism how differential operators annihilate the integrand of the integrals discussed above. The reason is two-fold: firstly, it would be interesting to understand in more detail how the distributional term that freezes the loop integration is generated. Secondly we expect our differential equation method to apply to larger classes of integrals. We hope that this section will prove a useful starting point for such investigations.

We do not claim that we exhaust all differential equations for a given diagram, but we show that there is always at least one for all diagrams appearing in the one-loop and two-loop MHV amplitudes.

\section{A.1 Diagrams in the one-loop amplitude}

The form of one-loop MHV amplitude presented in [19] contains sum of two-mass-hard boxes and double pentagons with "magic numerator". As was mentioned above, for con- 
venience we use the pentagons with dashed lines, the difference being a parity odd piece that vanishes after integration (or is of order $\mathcal{O}\left(\mathrm{m}^{2}\right)$ in the massive regularization).

\section{A.1.1 Two-mass hard diagram}

The two-mass-hard boxes as integrals in momentum twistor space are ${ }^{8}$

$$
I_{2 m h}=\frac{N}{(A B 12)(A B 23)(A B 34)(A B i i+1)}, \quad \text { where } \quad N=(1234)(23 i i+1) .
$$

This integrand is annihilated trivially by the first order differential operators $O_{21}, O_{34}$, $O_{i i+1}, O_{i+1 i}$ and also $O_{k j}$ for all $k$ and $j=5, \ldots i-1, i+2 \ldots n$.

We are interested in obtaining non-trivial second order differential equations for $I_{2 m h}^{i}$. Applying the operator $O_{24}$ on the un-normalised integrand $I_{2 m h} / N$ we get

$$
O_{24} \frac{1}{(A B 12)(A B 23)(A B 34)(A B i i+1)}=\frac{1}{(A B 12)(A B 34)^{2}(A B i i+1)},
$$

and this is trivially annihilated by $O_{12}$ and $O_{43}$. Similarly, we can start with the operator $O_{31}$ and then apply $O_{12}$ and $O_{43}$. As a result, we get four differential equations ${ }^{9}$

$$
\tilde{O}_{1224} I_{2 m h}=\tilde{O}_{4324} I_{2 m h}=\tilde{O}_{1231} I_{2 m h}=\tilde{O}_{4331} I_{2 m h}=0,
$$

where

$$
\tilde{O}_{i j k l}=N O_{i j} O_{k l} N^{-1} .
$$

In the boundary case of $i=4$ or $i=n$ (which corresponds to a one-mass diagram) we cannot use both "directions" of the derivatives. So in the first case we use only $O_{31}$ to create a massive corner, while in the second case only $O_{24}$. There is a special case of $n=4$ which corresponds to the zero-mass diagram. In this case we cannot use our construction. However, this diagram itself represents the one-loop MHV amplitude and we will see at the end of this subsection that there exist differential operators that annihilate naively all MHV one-loop integrands (including the $n=4$ case).

For reasons that will become clear later, it will be quite useful also to find a differential operator acting only on a limited number of twistors. Suppose that the only operators we want to use are $O_{k 2}, O_{k 3}, O_{n 1}, O_{54}$ for any $k$. Even in that case there exists a second-order operator, although somewhat complicated,

$$
\left[\tilde{O}_{4354}-\frac{(2345)}{(1234)} \tilde{O}_{1223}-\frac{(2456)}{(2356)} \tilde{O}_{54}+\frac{(1356)(2345)}{(1234)(2356)} \tilde{O}_{23}\right] I_{2 m h}=0
$$

and also its flipped version. It is hard to find an explanation for this operator (like we did for other operators above), but its existence is important for the discussion of the two-loop double boxes.

\footnotetext{
${ }^{8}$ We changed the labels from $i \rightarrow i+1$ w.r.t. to the main text.

${ }^{9}$ Note that we do not include the second order operators that include $O_{21}$ and $O_{34}$ as first order pieces.
} 


\section{A.1.2 Pentagon diagram}

The pentagon integrals in momentum twistor space are

$$
I_{\text {pent }}^{i j}=\frac{N(A B(123) \cap(i-1 i i+1))}{(A B 12)(A B 23)(A B i-1 i)(A B i i+1)(A B j j+1)},
$$

where $N=(2 i j j+1)$. The strategy is very similar to the two-mass hard case. We can find many trivial first order operators that annihilate the integrand, and we can use again the same trick of creating a massive corner. Here we have even more freedom in the choice which propagator we want to kill, e.g. we can first apply the operator $O_{13}$, yielding

$$
\begin{aligned}
O_{13} \frac{(A B(123)}{} & \cap(i-1 i i+1)) \\
= & \frac{(A B(123) \cap(i-1 i i+1))}{(A B 23)^{2}(A B i-1 i)(A B i i+1)(A B j j+1)},
\end{aligned}
$$

which is annihilated by the operator $O_{32}$. Finally, we can find four operators ${ }^{10}$ that nontrivially annihilate $I_{\text {pent }}^{i j}$,

$$
\tilde{O}_{3213} I_{\text {pent }}^{i j}=\tilde{O}_{1231} I_{\text {pent }}^{i j}=0
$$

and the same for $(1,2,3) \rightarrow(i+1, i, i-1)$. We see that the operators act separately just on the left or right region.

Now, something interesting happens when $i=4$, i.e. when the top corner is massless. Then both regions $(1,2,3) \rightarrow(i+1, i, i-1)$ overlap and the equations we wrote are not valid anymore. However, the numerator saves the situation and helps us to find another differential equation. First, let us apply the operator $O_{23}$,

$$
\begin{aligned}
O_{23} & \frac{(A B(123) \cap(345))}{(A B 12)(A B 23)(A B 34)(A B 45)(A B j j+1)}= \\
& =\frac{(A B(123) \cap(245))}{(A B 12)(A B 23)(A B 34)(A B 45)(A B j j+1)}-\frac{(A B 24)(A B(123) \cap(345))}{(A B 12)(A B 23)(A B 34)^{2}(A B 45)(A B j j+1)} \\
& =\frac{(1234)}{(A B 12)(A B 34)(A B j j+1)},
\end{aligned}
$$

where we used $(A B(123) \cap(234))=(A B 23)(1234)$. The term we obtained is annihilated by $O_{12}$ and $O_{34}$. Similarly, we can start with the operator $O_{43}$ and repeat the above calculation. In total we get four differential equations,

$$
\tilde{O}_{1223} I_{\text {pent }}^{4 j}=\tilde{O}_{3423} I_{\text {pent }}^{4 j}=\tilde{O}_{5443} I_{\text {pent }}^{4 j}=\tilde{O}_{3243} I_{\text {pent }}^{4 j}=0 .
$$

Here there are no boundary cases for $j=5$ or $j=n$.

In addition to these operators, we can also find two more special operators that annihilate the integrand of the pentagon. Like in the two-mass hard case they also contain only a restricted number of derivatives, but their forms are much nicer,

$$
\left[\sum_{k=2}^{i-2} \tilde{O}_{k i-11 k}+O_{1 i-1}\right] I_{\text {pent }}^{i, j}=\left[\sum_{k=4}^{i} \tilde{O}_{k 3 i+1 k}+O_{3 i+1}\right] I_{\text {pent }}^{i, j}=0 .
$$

\footnotetext{
${ }^{10}$ For the boundary case when $j=i+1$ or $j=n$ some operators are not present.
} 
We can see that two of equations for $i=4$ were exactly of this type.

We completed the picture of differential operators for all diagrams in one-loop MHV amplitude. Of course, the one-loop MHV amplitude is known analytically, but this result will be extremely useful in analyzing the diagrams contributing to the two-loop MHV amplitude.

\section{A.1.3 Differential equations for the one-loop MHV amplitude}

So far, we analyzed the one-loop amplitude giving the equations for all diagrams contributing to it. However, there exists an equation for the integrand of the whole amplitude. In fact, for the $n$-point amplitude there exist $n^{2}$ differential equations of the form

$$
Q_{i j} I_{M H V}^{1-l o o p}=0 \quad i, j=1, \ldots n
$$

where

$$
Q_{i j}=\sum_{k=1}^{n} O_{i k} O_{k j}-(n-2) O_{i j}
$$

Note that the structure of operators is quite similar to that which we found for the pentagons at the end of the last subsection.

\section{A.2 Diagrams in the two-loop amplitude}

We start with the form of two-loop MHV amplitude given in [19] and reviewed in section 2. It contains three topologies: double-boxes, penta-boxes and double-pentagons.

We will analyze each topology separately. Naively, they look like one-loop diagrams (two-mass hard integrals and pentagons) glued together, and we will see that in some sense they really are.

We want to find a second-order differential equation for the integrands of these diagrams, keeping in mind our motivation that acting with the correct second-order differential operator on a two-loop diagram can decrease the loop level by one). Therefore, it would be very natural to act just on a one-loop sub-diagram, which is exactly what we are going to do. We will see that there is close link with the operators found in the last subsection.

\section{A.2.1 Double-boxes}

The integrand for the general double-box appearing in the amplitude

$$
I_{\text {double-box }}^{i}=\frac{(1234)(i-1 i i+1 i+2)(23 i i+1)}{(A B 12)(A B 23)(A B 34)(A B C D)(C D i-1 i)(C D i i+1)(C D i+1 i+2)},
$$

can be rewritten in a very suggestive form

$$
\frac{(1234)(23 i i+1)}{(A B 12)(A B 23)(A B 34)(A B i i+1)} \cdot \frac{(i-1 i i+1 i+2)(A B i i+1)}{(A B C D)(C D i-1 i)(C D i i+1)(C D i+1 i+2)} .
$$

We see that the double box can be written in a factorised form as a product of two-mass hard diagrams (note that they are properly normalised), where the right CD sub-diagram contains twistors A,B as external, but the left AB sub-diagram depends only on external 
twistors. If $5<i<n-1$ (both internal corners are massive), then there is no overlap between $\mathrm{AB}$ and $\mathrm{CD}$ sub-diagrams and the $\mathrm{AB}$ part is annihilated by the same operators as the normal 2-mass-hard integrand,

$$
\tilde{O}_{1224} I_{2 b o x}=\tilde{O}_{4324} I_{2 b o x}=\tilde{O}_{1231} I_{2 b o x}=\tilde{O}_{4331} I_{2 b o x}=0
$$

where here by the normalisation $N$ we mean the normalisation of left sub-diagram $N=$ (1234)(23i $i+1)$. If $i=4,5, n-1, n$ just half of the operators survive (we still have one massive corner).

For $n=4,5,6$ we have diagrams that do not contain any massive corners. However, the diagrams for $n=5$ and for $n=6, i=5$ satisfy differential equations and here we use the complicated forms that we found in the end of the discussion of the two-mass hard boxes. Thanks to restrictions we made on the first-order operators for the construction of the second-order operator, these operators act just on one half of the double-box integrand even if the indices overlap (which exactly happens for $i=5$ or $i=n-1$ ). The only constraint is that at least one external line (which can be massless and just on one side of the diagram) must be attached at the point were the two one-loop diagrams are glued together. Then the equations are exactly the same as we found for the two-mass hard case, since we act on just half of the diagram.

\section{A.2.2 Pentagon-boxes}

As in the previous case, we can write the integrand for the pentagon-box appearing in the two-loop MHV amplitude,

$$
I_{n ; i, j}^{\text {penta-box }}=\frac{(A B(123) \cap(i-1 i i+1))(2 i j j+1)(j-1 j j+1 j+2)}{(A B 12)(A B 23)(A B i-1 i)(A B i i+1)(A B C D)(C D j-1 j)(C D j j+1)(C D j+1 j+2)},
$$

in a factorised form,

$$
\begin{aligned}
& \frac{(A B(123) \cap(i-1 i i+1))(2 i j j+1)}{(A B 12)(A B 23)(A B i-1 i)(A B i i+1)(A B j j+1)} \cdot \frac{(j-1 j j+1 j+2)(A B j j+1)}{(A B C D)(C D j-1 j)(C D j j+1)(C D j+1 j+2)} \\
& =\frac{(A B(123) \cap(i-1 i i+1))(C D 2 j)}{(A B 12)(A B 23)(A B i-1 i)(A B i i+1)(A B C D)} \cdot \frac{(2 i j j+1)(j-1 j j+1 j+2)}{(C D 2 j)(C D j-1 j)(C D j j+1)(C D j+1 j+2)} .
\end{aligned}
$$

We see that there are two choices, either the pentagon or the two-mass-hard is the "proper" one-loop subdiagram.

If we use the first choice, then the the two-loop diagram is annihilated by all operators that annihilate its pentagon one-loop sub-diagram, and the same is true for the second choice, where we use the differential operators for the two-mass-hard diagram. If the indices do not overlap, we can just act with the operators that we have for pentagons, resp. two-mass hard and we annihilate the integrand. In the special case of $j=i+1$ or $j=n-1$ we would repeat the same discussion as in the case of double-boxes. Here we use the more complicated forms that we derived in the end of the two-mass hard and pentagon sections that can be used even if the indices overlap. Therefore, all pentagon-box integrands satisfy second-order differential equations.

\section{A.2.3 Double-pentagons}

Finally, we can write the integrand for the double-pentagons,

$$
\frac{(A B(123) \cap(\ell-1 \ell \ell+1))(C D(i-1 i i+1) \cap(j-1 j j+1))(2 \ell i j)}{(A B 12)(A B 23)(A B \ell-1 \ell)(A B \ell \ell+1)(A B C D)(C D i-1 i)(C D i i+1)(C D j-1 j)(C D j j+1)},
$$


in a factorised form,

$$
\frac{(A B(123) \cap(\ell-1 \ell \ell+1))(2 \ell i j)}{(A B 12)(A B 23)(A B \ell-1 \ell)(A B \ell \ell+1)(A B i j)} \frac{(C D(i-1 i i+1) \cap(j-1 j j+1))(A B i j)}{(A B C D)(C D i-1 i)(C D i i+1)(C D j-1 j)(C D j j+1)} .
$$

We again see two correctly normalised pentagons, and one of them is a proper pentagon on which we can apply differential operators. The discussion would be identical to the previous cases, one just uses the operators found in the pentagon section which are guaranteed to annihilate also the double-pentagon integrand.

\section{B Five-point pentagon and penta-box integrals}

As an explicit example of the differential equations for regulated integrals of section 3, we give the result for the five-point pentagon and penta-box integrals at one and two loops. Let us start with the one-loop pentagon integral. Without loss of generality, we choose it in the orientation

$$
F_{5 ; 5,1,3}^{\text {pent }}=\int \frac{d^{4} Z_{A B}}{i \pi^{2}} \frac{(2345)(4512)(A B 13)}{(A B 51)(A B 12)(A B 23)(A B 34)(A B 45)},
$$

and we recall the reader that because of infrared divergences we also include $+m^{2}$ terms in the definition of the propagators, see section 2. Introducing Feynman parameters, we obtain

$$
F_{5 ; 5,1,3}^{\text {pent }}=-2 x_{35}^{2} x_{25}^{2} x_{14}^{2} \int_{0}^{\infty} \frac{d \alpha_{i} \alpha_{5} \delta\left(\sum_{i=1}^{5} \alpha_{i}-1\right)}{\left(\sum_{i=1}^{5} \alpha_{i} \alpha_{i+2} x_{i, i+2}^{2}+m^{2}\right)^{3}}+\mathcal{O}\left(m^{2}\right) .
$$

The small $\mathrm{m}^{2}$ expansion of this integral can be conveniently obtained using Mellin-Barnes methods. The answer depends on three variables, $y_{1}=x_{35}^{2} / x_{13}^{2}, y_{2}=x_{25}^{2} / x_{24}^{2}, y_{3}=x_{14}^{2} / \mathrm{m}^{2}$, and can be written as

$$
F_{5 ; 5,1,3}^{\text {pent }}=-\frac{1}{2} \log ^{2}\left(y_{1} y_{2} y_{3}\right)-2 \operatorname{Li}_{2}\left(1-y_{1}\right)-2 \operatorname{Li}_{2}\left(1-y_{2}\right)+\frac{\pi^{2}}{6}+\mathcal{O}\left(m^{2}\right) .
$$

We can also compute the corresponding two-loop penta-box integral. In the nomenclature of section 2 , it is

$$
F_{5 ; 5,1,3}^{\text {penta-box }}=\int \frac{d^{4} Z_{A B} d^{4} Z_{C D}\left(i \pi^{2}\right)^{-2} N(C D 13)}{(A B 34)(A B 45)(A B 51)(A B C D)(C D 51)(C D 12)(C D 23)(C D 34)},
$$

where $N=(3451)(2345)(4512)$. It evaluates to

$$
F_{5 ; 5,1,3}^{\text {penta-box }}=h\left(y_{1} y_{2} y_{3}\right)-2 H_{0}\left(y_{1} y_{2} y_{3}\right)\left[f\left(y_{1}\right)+f\left(y_{2}\right)\right]+\left[g\left(y_{1}\right)+g\left(y_{2}\right)\right]+\mathcal{O}\left(m^{2}\right),
$$

where

$$
\begin{aligned}
& h(x)=-\frac{7}{40} \pi^{4}+2 \zeta_{3} H_{0}(x)-\frac{1}{2} \pi^{2} H_{0,0}(x)-H_{0,0,0,0}(x), \\
& g(x)=-\frac{2}{3} H_{2}(x)+2 H_{0}(x) H_{2,0}(x)-4 H_{3,0}(x)-2 H_{2,0,0}(x)-4 H_{2,1,0}(x), \\
& f(x)=H_{0,1,0}(x) .
\end{aligned}
$$


The integrals satisfy

$$
y_{1} \frac{\partial}{\partial y_{1}} y_{2} \frac{\partial}{\partial y_{2}} F_{5 ; 5,1,3}^{\text {pent }}=-1+\mathcal{O}\left(m^{2}\right)
$$

and

$$
y_{1} \frac{\partial}{\partial y_{1}} y_{2} \frac{\partial}{\partial y_{2}} F_{5 ; 5,1,3}^{\text {penta-box }}=F_{5 ; 5,1,3}^{\text {pent }}+\mathcal{O}\left(m^{2}\right) .
$$

One can check that equations (3.79) and (3.81) are indeed satisfied.

Open Access. This article is distributed under the terms of the Creative Commons Attribution Noncommercial License which permits any noncommercial use, distribution, and reproduction in any medium, provided the original author(s) and source are credited.

\section{References}

[1] J.M. Drummond, J. Henn, G.P. Korchemsky and E. Sokatchev, Dual superconformal symmetry of scattering amplitudes in $N=4$ super-Yang-Mills theory,

Nucl. Phys. B 828 (2010) 317 [arXiv:0807.1095] [SPIRES].

[2] A. Brandhuber, P. Heslop and G. Travaglini, A note on dual superconformal symmetry of the $N=4$ super Yang-Mills S-matrix, Phys. Rev. D 78 (2008) 125005 [arXiv:0807.4097] [SPIRES].

[3] J.M. Drummond and J.M. Henn, All tree-level amplitudes in $N=4 S Y M$, JHEP 04 (2009) 018 [arXiv:0808.2475] [SPIRES].

[4] J.M. Drummond, J.M. Henn and J. Plefka, Yangian symmetry of scattering amplitudes in $N=4$ super Yang-Mills theory, JHEP 05 (2009) 046 [arXiv:0902.2987] [SPIRES].

[5] J.M. Drummond, J. Henn, G.P. Korchemsky and E. Sokatchev, Conformal Ward identities for Wilson loops and a test of the duality with gluon amplitudes,

Nucl. Phys. B 826 (2010) 337 [arXiv:0712.1223] [SPIRES].

[6] C. Anastasiou, Z. Bern, L.J. Dixon and D.A. Kosower, Planar amplitudes in maximally supersymmetric Yang-Mills theory, Phys. Rev. Lett. 91 (2003) 251602 [hep-th/0309040] [SPIRES].

[7] Z. Bern, L.J. Dixon and V.A. Smirnov, Iteration of planar amplitudes in maximally supersymmetric Yang-Mills theory at three loops and beyond, Phys. Rev. D 72 (2005) 085001 [hep-th/0505205] [SPIRES].

[8] J.M. Drummond, J. Henn, G.P. Korchemsky and E. Sokatchev, Hexagon Wilson loop = six-gluon MHV amplitude, Nucl. Phys. B 815 (2009) 142 [arXiv:0803.1466] [SPIRES].

[9] Z. Bern et al., The two-loop six-gluon MHV amplitude in maximally supersymmetric Yang-Mills theory, Phys. Rev. D 78 (2008) 045007 [arXiv:0803.1465] [SPIRES].

[10] L.F. Alday, J.M. Henn, J. Plefka and T. Schuster, Scattering into the fifth dimension of $N=4$ super Yang-Mills, JHEP 01 (2010) 077 [arXiv:0908.0684] [SPIRES].

[11] L.F. Alday and J.M. Maldacena, Gluon scattering amplitudes at strong coupling, JHEP 06 (2007) 064 [arXiv: 0705.0303] [SPIRES].

[12] J.M. Henn, S.G. Naculich, H.J. Schnitzer and M. Spradlin, Higgs-regularized three-loop four-gluon amplitude in $N=4$ SYM: exponentiation and Regge limits, JHEP 04 (2010) 038 [arXiv: 1001.1358] [SPIRES]. 
[13] R.H. Boels, No triangles on the moduli space of maximally supersymmetric gauge theory, JHEP 05 (2010) 046 [arXiv: 1003.2989] [SPIRES].

[14] Z. Bern, J.J. Carrasco, T. Dennen, Y.-t. Huang and H. Ita, Generalized unitarity and six-dimensional helicity, arXiv:1010.0494 [SPIRES].

[15] A. Sever and P. Vieira, Symmetries of the N=4 SYM S-matrix, arXiv:0908.2437 [SPIRES].

[16] T. Bargheer, N. Beisert, W. Galleas, F. Loebbert and T. McLoughlin, Exacting $N=4$ superconformal symmetry, JHEP 11 (2009) 056 [arXiv: 0905.3738] [SPIRES].

[17] G.P. Korchemsky and E. Sokatchev, Symmetries and analytic properties of scattering amplitudes in $N=4$ SYM theory, Nucl. Phys. B 832 (2010) 1 [arXiv:0906.1737] [SPIRES].

[18] N. Beisert, J. Henn, T. McLoughlin and J. Plefka, One-loop superconformal and yangian symmetries of scattering amplitudes in $N=4$ super Yang-Mills, JHEP 04 (2010) 085 [arXiv: 1002.1733] [SPIRES].

[19] N. Arkani-Hamed, J.L. Bourjaily, F. Cachazo, S. Caron-Huot and J. Trnka, The all-loop integrand for scattering amplitudes in planar $N=4$ SYM, JHEP 01 (2011) 041 [arXiv: 1008.2958] [SPIRES].

[20] R.H. Boels, On BCFW shifts of integrands and integrals, JHEP 11 (2010) 113 [arXiv:1008.3101] [SPIRES].

[21] N. Arkani-Hamed, F. Cachazo and C. Cheung, The grassmannian origin of dual superconformal invariance, JHEP 03 (2010) 036 [arXiv:0909.0483] [SPIRES].

[22] L.J. Mason and D. Skinner, Dual superconformal invariance, momentum twistors and grassmannians, JHEP 11 (2009) 045 [arXiv: 0909.0250] [SPIRES].

[23] J.M. Drummond and L. Ferro, The yangian origin of the grassmannian integral, JHEP 12 (2010) 010 [arXiv: 1002 .4622] [SPIRES].

[24] G.P. Korchemsky and E. Sokatchev, Superconformal invariants for scattering amplitudes in $N=4$ SYM theory, Nucl. Phys. B $\mathbf{8 3 9}$ (2010) 377 [arXiv:1002.4625] [SPIRES].

[25] J.M. Drummond, J. Henn, V.A. Smirnov and E. Sokatchev, Magic identities for conformal four-point integrals, JHEP 01 (2007) 064 [hep-th/0607160] [SPIRES].

[26] L.F. Alday and R. Roiban, Scattering amplitudes, Wilson loops and the string/gauge theory correspondence, Phys. Rept. 468 (2008) 153 [arXiv:0807.1889] [SPIRES].

[27] J.M. Henn, Duality between Wilson loops and gluon amplitudes, Fortsch. Phys. 57 (2009) 729 [arXiv:0903.0522] [SPIRES].

[28] L.J. Mason and D. Skinner, The complete planar S-matrix of $N=4$ SYM as a Wilson loop in twistor space, JHEP 12 (2010) 018 [arXiv: 1009.2225] [SPIRES].

[29] S. Caron-Huot, Notes on the scattering amplitude/Wilson loop duality, arXiv:1010.1167 [SPIRES].

[30] A.B. Goncharov, M. Spradlin, C. Vergu and A. Volovich, Classical polylogarithms for amplitudes and Wilson loops, Phys. Rev. Lett. 105 (2010) 151605 [arXiv:1006.5703] [SPIRES].

[31] V. Del Duca, C. Duhr and V.A. Smirnov, The two-loop hexagon Wilson loop in $N=4 S Y M$, JHEP 05 (2010) 084 [arXiv: 1003.1702] [SPIRES]. 
[32] V. Del Duca, C. Duhr and V.A. Smirnov, A two-loop octagon Wilson loop in $N=4 S Y M$, JHEP 09 (2010) 015 [arXiv: 1006.4127] [SPIRES].

[33] P. Heslop and V.V. Khoze, Analytic results for MHV Wilson loops, JHEP 11 (2010) 035 [arXiv: 1007.1805] [SPIRES].

[34] J.M. Henn, S.G. Naculich, H.J. Schnitzer and M. Spradlin, More loops and legs in Higgs-regulated $N=4$ SYM amplitudes, JHEP 08 (2010) 002 [arXiv:1004.5381] [SPIRES].

[35] D.A. Kosower, R. Roiban and C. Vergu, The six-point NMHV amplitude in maximally supersymmetric Yang-Mills theory, Phys. Rev. D 83 (2011) 065018 [arXiv:1009.1376] [SPIRES].

[36] L.F. Alday, Some analytic results for two-loop scattering amplitudes, arXiv:1009.1110 [SPIRES].

[37] L.F. Alday, B. Eden, G.P. Korchemsky, J. Maldacena and E. Sokatchev, From correlation functions to Wilson loops, arXiv:1007.3243 [SPIRES].

[38] B. Eden, G.P. Korchemsky and E. Sokatchev, More on the duality correlators/amplitudes, arXiv: 1009. 2488 [SPIRES].

[39] A.V. Kotikov, Differential equations method: New technique for massive Feynman diagrams calculation, Phys. Lett. B 254 (1991) 158.

[40] A.V. Kotikov, Differential equation method: the calculation of $N$ point Feynman diagrams, Phys. Lett. B 267 (1991) 123.

[41] T. Gehrmann and E. Remiddi, Differential equations for two-loop four-point functions, Nucl. Phys. B 580 (2000) 485 [hep-ph/9912329] [SPIRES].

[42] V. Smirnov, Feynman integral calculus, Springer, U.S.A. (2006).

[43] J.M. Drummond and J.M. Henn, Simple loop integrals and amplitudes in $N=4 S Y M$, arXiv: 1008.2965 [SPIRES].

[44] A. Hodges, Eliminating spurious poles from gauge-theoretic amplitudes, arXiv:0905.1473 [SPIRES].

[45] A. Hodges, The box integrals in momentum-twistor geometry, arXiv:1004.3323 [SPIRES].

[46] L. Mason and D. Skinner, Amplitudes at weak coupling as polytopes in $A d S_{5}$, J. Phys. A 44 (2011) 135401 [arXiv:1004.3498] [SPIRES].

[47] Z. Bern, L.J. Dixon, D.C. Dunbar and D.A. Kosower, One-loop n-point gauge theory amplitudes, unitarity and collinear limits, Nucl. Phys. B 425 (1994) 217 [hep-ph/9403226] [SPIRES].

[48] Z. Bern, L.J. Dixon, D.C. Dunbar and D.A. Kosower, One-loop n-point gauge theory amplitudes, unitarity and collinear limits, Nucl. Phys. B 425 (1994) 217 [hep-ph/9403226] [SPIRES].

[49] N.I. Usyukina and A.I. Davydychev, Exact results for three and four point ladder diagrams with an arbitrary number of rungs, Phys. Lett. B 305 (1993) 136.

[50] A.P. Isaev, Multi-loop Feynman integrals and conformal quantum mechanics, Nucl. Phys. B 662 (2003) 461 [hep-th/0303056] [SPIRES].

[51] N. Arkani-Hamed, J. Bourjaily, F. Cachazo, S. Caron-Hout and J. Trnka, Local integrals for planar scattering amplitudes, arXiv:1012.6032 [SPIRES]. 
[52] E. D'Hoker and D.Z. Freedman, Supersymmetric gauge theories and the AdS/CFT correspondence, in Strings, branes and extra dimensions, S.S. Gubser and J.D. Lykken eds., World Scientific, Singapore (2004), hep-th/0201253 [SPIRES].

[53] E. Remiddi and J.A.M. Vermaseren, Harmonic polylogarithms, Int. J. Mod. Phys. A 15 (2000) 725 [hep-ph/9905237] [SPIRES].

[54] T. Gehrmann and E. Remiddi, Two-loop master integrals for $\gamma^{*} \rightarrow 3$ jets: the planar topologies, Nucl. Phys. B 601 (2001) 248 [hep-ph/0008287] [SPIRES].

[55] D.J. Broadhurst and A.I. Davydychev, Exponential suppression with four legs and an infinity of loops, Nucl. Phys. Proc. Suppl. 205-206 (2010) 326 [arXiv:1007.0237] [SPIRES].

[56] C. Anastasiou, S. Beerli, S. Bucherer, A. Daleo and Z. Kunszt, Two-loop amplitudes and master integrals for the production of a Higgs boson via a massive quark and a scalar-quark loop, JHEP 01 (2007) 082 [hep-ph/0611236] [SPIRES]. 